\title{
Overexpression of TMEFF1 in Endometrial Carcinoma and the Mechanism Underlying its Promotion of Malignant Behavior in Cancer Cells
}

\author{
Xin $\mathrm{Nie}^{1,2^{*}}$, Lingling Gao ${ }^{1,2^{*}}$, Mingjun Zheng ${ }^{3}$, Caixia Wang ${ }^{4}$, Shuang Wang ${ }^{1,2}$, Xiao $\mathrm{Li}^{1,2}$, Yue Qi1,2, \\ Liancheng Zhu ${ }^{1,2}$, Juanjuan Liu ${ }^{1,2}$, Bei Lin ${ }^{1,2}$ \\ 1. Shengjing Hospital of China Medical University, Department of Obstetrics and Gynecology, Shenyang, China \\ 2. Key Laboratory of Maternal-Fetal Medicine of Liaoning Province, Key Laboratory of Obstetrics and Gynecology of Higher Education of Liaoning Province, \\ Shenyang, China. \\ 3. University Hospital, LMU Munich, Department of Obstetrics and Gynecology, Munich, Germany \\ 4. West China Second University Hospital, Sichuan University, Department of Obstetrics and Gynecology, Sichuan, China \\ ${ }^{*}$ Co-first authors.
}

$\triangle$ Corresponding author: Bei Lin, E-mail: linbei88@hotmail.com; Shengjing Hospital of China Medical University, 36 Sanhao Road, Heping District, Shenyang 110004, China. Cell: 86-18940256796; Tel(O): 86-96615-41511.

(C) The author(s). This is an open access article distributed under the terms of the Creative Commons Attribution License (https://creativecommons.org/licenses/by/4.0/). See http://ivyspring.com/terms for full terms and conditions.

Received: 2021.01.22; Accepted: 2021.07.29; Published: 2021.07.31

\begin{abstract}
Background: Although tomoregulin-1 (TMEFFI) is involved in embryonic development and central nervous system regulation and is a cancer suppressor gene in brain cancers, its role in endometrial carcinoma remains unclear.

Methods: The expression and prognostic value of TMEFF1 were analyzed by bioinformatics methods and immunohistochemistry. An endometrial carcinoma cell line with low expression of TMEFF1 was constructed. Scratch and Transwell assays were used to determine the effect of TMEFFI on cell invasion and migration. Changes in key proteins in the MAPK and PI3K/AKT signaling pathways and in epithelial-mesenchymal transition (EMT)-related proteins were analyzed using western blot. Chromatin immunoprecipitation assay (ChIP) was performed to identify whether the TMEFFI promoter region binds to the transcription factor $\mathrm{p} 53$.

Results: TMEFF1 was significantly upregulated in endometrial carcinoma, was closely associated with FIGO stage $(P=0.021)$ and lymph node metastasis $(P=0.029)$, and was an independent risk factor for prognosis $(P=0.044)$. Gene Ontology (GO) and Kyoto Encyclopedia of Genes and Genomes (KEGG) pathway analyses showed that TMEFFI and its related genes are involved in the cell cycle, regulation of mitosis, epigenetics, neural development, cell biological signal transduction and some key signal pathways. We also identified kinases, microRNAs and a transcription factor network related to TMEFF1 and the effect of TMEFF1 mutation on prognosis. In vitro knockdown of TMEFFI significantly inhibited cell invasion and migration. Knockdown of TMEFFI inhibited Epithelial-mesenchymal transition (EMT) and activation of the MAPK and PI3K/AKT pathways. However, the transcription factor p53 was not found to regulate the TMEFFI gene.

Conclusion: TMEFF1 plays an important role in endometrial carcinoma and may thus be a potential anticancer therapeutic target for endometrial carcinoma.
\end{abstract}

Key words: TMEFF1, endometrial carcinoma, EMT, MAPK, metastasis

\section{Introduction}

Endometrial carcinoma is one of the common malignant cancers of the female reproductive system. In developed countries, the mortality rate is second only to ovarian cancer, and its incidence is increasing year by year [1]. Endometrial carcinoma is usually divided into two major clinical pathological types: type I (estrogen-dependent) endometrial carcinoma, accounting for $80 \%$ of endometrial carcinomas; and type II (nonestrogen-dependent) endometrial carcinoma, including serous cancer and clear cell carcinoma, which usually occurs in the atrophic endometrium after menopause; type II has worse 
prognosis than type I [2]. There are few methods for clinically treating endometrial carcinoma. In addition, the lesions cannot be completely removed in late-stage patients and the long-term recurrence rate is high. Moreover, the sensitivity of radiotherapy and chemotherapy is poor, with considerable toxicity, multiple adverse effects, and poor efficacy. Therefore, it is vital to identify new preventive methods [3].

Cancer/testis antigens (CTAs) are cancerassociated antigens that are mainly expressed in cells of the testis and embryonic tissues and lowly or not expressed in other normal tissues [4,5]. Because these antigens are expressed in different types of malignant cells, they are an antigenic target for the early detection of cancers and efficacy of cancer immunotherapy [6]. TMEFF1 is the $120^{\text {th }}$ member of the CTA family [7]. In early studies, TMEFF1 was a cancer suppressor in brain cancers $[8,9]$. Work showed that TMEFF1 was highly expressed in ovarian cancer tissues, promoted the malignant behavior of cancers, and was closely associated with the occurrence and progression of malignant cancers [10]. A number of questions remain, such as the expression of TMEFF1 in endometrial carcinoma and whether it plays a role in suppressing or promoting cancer. There has been no research into TMEFF1 in endometrial carcinoma. Accordingly, we use several bioinformatics databases to analyze the expression of TMEFF1 in endometrial carcinoma and functional regulatory networks. Subsequently, we examined the expression of TMEFF1 in endometrial clinical samples and analyzed its clinical significance. We additionally investigated the effect of TMEFF1 on the malignant behavior of endometrial carcinoma and its mechanism, providing a theoretical basis for the early diagnosis and immunotherapy of endometrial carcinoma.

\section{Methods}

\section{Oncomine database analysis}

The Oncomine database (http://www. oncomine.org) [11] is an oncogene database and integrated data mining platform that enables analysis of differential expression in clinically common cancer tissues and their corresponding normal tissues.

\section{UALCAN analysis}

UALCAN (http://ualcan.path.uab.edu) [12] is an effective online analysis tool and cancer database that enables the user to analyze data according to cancer stage, tumor grade, and patient race and weight, as well as the relative expression of genes of interest and clinicopathological characteristics in tumor and normal samples. It can also be used to estimate the impact of gene expression levels and clinicopathological characteristics on patient survival.

\section{LinkedOmics analysis}

The LinkedOmics database (http://www. linkedomics.org/login.php) [13, 14] is an online platform for analyzing multiomics data and clinical data. The LinkFinder module was used to study the differentially expressed genes related to TMEFF1 in the TCGA uterine corpus endometrial carcinoma (UCEC) dataset, and Pearson correlation coefficients were determined and used to statistically analyze the results. Volcano plots, heatmaps and scatter plots were constructed for each differentially expressed gene related to TMEFF1. The LinkInterpreter module first standardizes and sorts all differentially expressed genes and then enriches and analyzes the signal pathways and network regulation of the genes of interest. The cutoff used was FDR $<0.05$, and 500 simulations were performed.

\section{Metascape analysis}

Metascape (http://metascape.org)[15] is a tool for gene annotation and analysis that integrates multiple authoritative database resources, such as the GO project, KEGG, UniProt and DrugBank. In this study, Metascape was used to analyze the enrichment of TMEFF1 and its related differentially expressed genes in processes and pathways. Gene Ontology (GO) biological analysis provides results in terms of three categories: biological process, cellular component, and molecular function. The following were used as cutoff values: $P<0.01$, minimum count of 3 , and enrichment factor $>1.5$.

\section{GeneMANIA analysis}

GeneMANIA (http://www.genemania.org) [16] is an online platform that can predict gene function, analyze gene lists, and rank genes with clear functions. It is also used to construct protein-protein interaction (PPI) networks, protein-DNA interaction networks, and signaling pathways, determine gene and protein expression, and predict protein interaction domains and phenotypes, physiological and biochemical reactions and other processes. We used GeneMANIA to determine the proteins that interact with TMEFF1.

\section{STRING analysis}

The STRING database (https://string-db.org/) [17] is a database for searching for interactions between proteins, including both direct physical interactions between proteins and indirect functional correlations between proteins. We used the STRING database to analyze the proteins related to TMEFF1. 


\section{GEPIA analysis}

The GEPIA database (http://gepia.cancer-pku./ $\mathrm{cn} /$ index.html) [18] is an interactive web server used to integrate and analyze cancer expression profile data, including RNA sequencing expression data of tumor and normal samples from TCGA and GTEx. We used the "General" module of this online analysis tool to analyze the expression level of the TMEFF1 gene in various tumor tissues, and the "Expression DIY" module was used to analyze the expression of the TMEFF1 gene in UCEC. The screening conditions used in the "Expression DIY" module are as follows: gene: TMEFF1; dataset: UCEC; $\log 2 \mathrm{FC}$ cutoff $>1$; and $P<0.01$.

\section{cBioPortal analysis}

The data from the cBioPortal database (https:// www.cbioportal.org/) [19] come from TCGA, ICGC, GEO and other databases. The types of integrated genome data include somatic mutation, DNA copy number alteration (CNA), mRNA and miRNA expression, DNA methylation, protein abundance and phosphoprotein abundance data. cBioPortal can visualize genomic data of cancer research samples and genetic differences between samples as well as genes and pathways and can compare them with clinical results.

\section{Specimen source and clinical data}

Surgical paraffin-embedded pathology specimens were collected from 135 patients at the Department of Obstetrics and Gynecology, Shengjing Hospital affiliated to China Medical University, from 2004 to 2017. The pathological diagnosis of all histological sections was completed by a pathologist of Shengjing Hospital affiliated to China Medical University. There were 75 cases of endometrial carcinoma, 24 cases of endometrial atypical hyperplasia (8 of mild hyperplasia, 9 of moderate hyperplasia, and 8 of severe hyperplasia), and 36 cases of normal endometrium (21 in the secretory phase and 15 in the proliferative phase). Normal endometrial tissue was obtained from patients who had undergone total hysterectomy and bilateral adnexectomy or total hysterectomy due to cervical lesions, without fertility requirements. In addition, the atypical hyperplasia and normal endometrial tissue samples were from patients without uterine fibroids, chocolate cyst of the ovary, or other estrogendependent diseases.

The patients in the endometrial carcinoma group were 36 to 79 years old, with an average age of 60.0 years; the patients in the endometrial atypical hyperplasia group were 35 to 66 years old, with an average age of 47.5 years; and the patients in the normal endometrium group were 39 to 53 years old, with an average age of 44.1 years. There was no significant difference in the ages of each group $(P>0.05)$. The pathological types of endometrial carcinoma were as follows: 37 cases of endometrioid adenocarcinoma, 23 cases of serous papillary adenocarcinoma, 8 cases of clear cell carcinoma, and 7 cases of other pathological types (3 of mucinous carcinoma, 2 of squamous-cell carcinoma, and 2 of undifferentiated carcinoma). According to the pathological grades, 16 cases were highly differentiated, 29 were moderately differentiated, and 30 were poorly differentiated. According to the International Federation of Gynecology and Obstetrics (FIGO) 2009 staging, 50 cases were FIGO stage I, 6 were stage II, 16 were stage III, and 3 were stage IV. There were 14 cases of lymph node metastasis, 48 without lymph node metastasis, and 13 without lymph node dissection. There were 48 cases with $<1 / 2$ muscle infiltration and 27 with $\geq 1 / 2$ muscle infiltration. All patients had primary endometrial carcinoma, the clinical and pathological data were complete, and no radiotherapy or chemotherapy was performed before the surgery. Nine of the patients did not undergo estrogen receptor (ER) and progesterone receptor (PR) tests. The study was approved by the Ethics Committee of China Medical University. Written informed consent was obtained from all participants.

\section{Immunohistochemistry}

Endometrial tissue was serially sectioned at 5 $\mu \mathrm{m}$. The expression of TMEFF1 was determined using the immunohistochemical streptavidin-peroxidase ligation (SP) method. The staining method was carried out according to the SP kit manual (China Maxim). The positive control used normal human epididymal tissue and the negative control replaced the primary antibody with homologous IgG. The TMEFF1 antibody concentration was 1:70 (Bioss, China). Brown particles were evident in the cell membrane and/or cytoplasm and were classified according to color intensity as follows: 0 points, uncolored; 1, light yellow; 2, brown; and 3, dark brown. Positive cell rates < 5\%, 6-25\%, 26-50\%, $51-75 \%$, and $>75 \%$ were scored as $0,1,2,3$, and 4 , respectively. The final score was obtained by multiplying the score of the two items mentioned above: $0-2$ was considered negative $(-), 3-4$ was $(+)$, 5-8 was $(++)$, and 9-12 was $(+++)$. Two observers participated in the scoring to control for errors.

\section{Cell culture and TMEFFI gene transfection}

The endometrial carcinoma cell lines Ishikawa and HEC1B were purchased from the Shanghai Cell 
Bank of the Chinese Academy of Sciences. The cells were routinely cultured in McCoy's 5A/DMEM medium containing $10 \%$ fetal calf serum (Gibco, USA) at $37{ }^{\circ} \mathrm{C}, 5 \% \mathrm{CO}_{2}$, and saturated humidity. Using siRNA transfection, cell lines with low expression of TMEFF1 and negative control cell lines were established and named Ishikawa-TMEFF1-siRNA, Ishikawa-NC, HEC-1-B-TMEFF1-siRNA and HEC-1B-NC. The blank control was named Ishikawa-Blank and HEC-1-B-Blank. Two siRNAs showed synergic effects on the knockdown of TMEFF1. The TMEFF1 siRNA sequence 1 (Genepharma, China) was as follows: sense, 5'-GCUCACUCAUGUUCUUAU UTT-3'; antisense: 5'- AAUAAGAACAUGAGUGAG CTT-3'. The TMEFF1 siRNA sequence 2 (Genepharma, China) was as follows: sense, 5'-TGCT GACTAAAGTCCGTCTTCTCACAG-3'; antisense: 5'TTTTGGCCACTGACTGACTGTGAGAACGGACTT TAGT-3'.

\section{Real-time quantitative PCR}

Total mRNA was extracted from endometrial carcinoma cells using TRIzol (TAKARA, Japan). The cDNA was then synthesized by reverse transcription of RNA using the Super Script III First-Strand Synthesis System RT-PCR kit (TAKARA). The real-time quantification procedure was performed via a two-step method: pre-denaturation at $95^{\circ} \mathrm{C}$ for $30 \mathrm{~s}$, followed by denaturation at $95{ }^{\circ} \mathrm{C}$ for $5 \mathrm{~s}$ and annealing at $60^{\circ} \mathrm{C}$ for $31 \mathrm{~s}$ for a total of 40 cycles. With GAPDH as an internal reference, real-time PCR amplification was performed using an AB17500 PCR machine to detect the $\mathrm{Ct}$ value of each template. After the amplification, a dissolution curve analysis was performed. The fold-changes were calculated using the $2-\Delta \Delta \mathrm{Ct}$ method. The experiment was repeated three times. The TMEFF1 primer (Sangon, Shanghai, China) was as follows: forward, 5'-TTGTTGGGAA AGAAAGATGA TGGA-3'; reverse, 5'-GATGCAGT AA CCATTGAGGT TTT-3'.

\section{Western blot}

Total protein was extracted from endometrial carcinoma cells and denatured. The protein was separated by $10 \%$ sodium dodecyl sulfatepolyacrylamide gel electrophoresis (SDS-PAGE) and transferred to a methanol-activated PVDF membrane. After blocking for $1 \mathrm{~h}$ using 5\% milk or BSA, the PVDF membrane was incubated with primary antibody at $4{ }^{\circ} \mathrm{C}$ overnight. The primary antibodies were as follows: anti-TMEFF1 antibody (Santa Cruz, 1:500); anti-PI3K p85 antibody (Cell Signaling Technology, 1:1000), anti-phospho-PI3K p85 antibody (Ser458) (Cell Signaling Technology, 1:1000), anti-AKT antibody (Cell Signaling Technology, 1:1000),
anti-phospho-AKT (Ser473) antibody (Cell Signaling Technology, 1:1000), anti-MEK1/2 antibody (Cell Signaling Technology, 1:1000), anti-phosphoMEK1/2 antibody (Cell Signaling Technology, 1:1000), anti-ERK1/2 antibody (Cell Signaling Technology, 1:1000), anti-phospho-ERK1/2 antibody (Cell Signaling Technology, 1:1000), anti-E-cadherin antibody (Cell Signaling Technology, 1:1000), antiVimentin antibody (Proteintech, 1:1000), anti-MMP2 antibody (Proteintech, 1:1000), anti-MMP9 antibody (Proteintech, 1:1000), and anti-GAPDH (Zhongsu Jinqiao, 1:2000). After washing with TBST, the membrane was incubated with secondary antibody (Nakasugi Jinqiao, 1:5000) for $2 \mathrm{~h}$ at room temperature. Protein bands were measured by ImageJ $1.31 \mathrm{v}$ and normalized to the expression levels of GAPDH.

\section{Scratch test}

Cells were seeded in a 6-well plate. After the cell density reached $90 \%$, the plate was gently scratched using a $100-\mu l$ pipette tip. The cells were then cultured in serum-free medium for 0 and $24 \mathrm{~h}$ and the width of the scratch was observed under a microscope. The experiment was repeated three times.

\section{Transwell assay}

Matrigel gel $(80 \mu \mathrm{l})$ was added to the upper chamber of a Transwell chamber (Corning Costar, USA) in a $37^{\circ} \mathrm{C}$ incubator for $5 \mathrm{~h}$. Then, $500 \mu \mathrm{l}$ of $10 \%$ fetal bovine serum culture medium was added to the lower chamber, and $200 \mu \mathrm{l}$ of a cell suspension in serum-free medium $\left(2 \times 10^{5}\right.$ cells $)$ was added to the upper chamber. The cells were incubated at $37^{\circ} \mathrm{C}$ for $48 \mathrm{~h}$. The upper chamber surface was gently wiped with a cotton swab and the cells in the lower chamber were fixed in $4 \%$ paraformaldehyde at room temperature for $25 \mathrm{~min}$ and stained with crystal violet for $25 \mathrm{~min}$. The numbers of cancer cells that infiltrated the filter membrane were counted under a microscope. Three replicate wells were set per well and the experiment was repeated three times.

\section{Chromatin immunoprecipitation (ChIP) assay}

Endometrial cancer cells (ISHIKAWA cells) were collected during the logarithmic growth phase. A commercially available kit (CST, 9004) and p53 antibody (CST, 2527s) were used for ChIP analysis, which was carried out according to the manufacturer's instructions. Real-time PCR was performed with the following primers: forward primer $\mathrm{F}$ covering the promoter region of TMEFF1: 5'-ATGGCTAGAGTCAGAACTTG-3'; reverse primer R: 5'-TGAGTCACGGAAGAGGTAA-3'. The negative control sequence was amplified by a primer that binds to the promoter region of the HCII gene, not within 
the TMEFF1 promoter or any other part of TMEFF1: forward primer $F$ of the negative control: 5'-TTATGTGGTGACCTCAAGAG-3'; reverse primer R: 5'-TGACGGTTACTGTGTTAGC-3'. The experiment was repeated three times.

\section{Statistical analysis}

The study data were analyzed with SPSS22.0 software. The measurement data were analyzed by single-factor analysis of variance. The counting data were analyzed by $x^{2}$ test and Fisher exact probability test. The survival curve was analyzed by KaplanMeier and log-rank tests. The relationship with patients was analyzed using Cox model. $P<0.05$ indicated that the difference was statistically significant.

\section{Results}

\section{Analysis of TMEFF 1 expression and prognosis in the Oncomine database, GEPIA database and UALCAN}

Data on TMEFF1 expression from studies in 377 different types of tumors were extracted from the Oncomine database. 23 studies showed significant differences in TMEFF1 mRNA levels. Among them, there were 15 studies with significantly higher expression of TMEFF1 mRNA and 8 studies with significantly lower expression of TMEFF1 mRNA. The expression of TMEFF1 mRNA was significantly increased in cervical cancer, colorectal cancer, esophageal cancer, gastric cancer, head and neck cancer, kidney cancer, lung cancer, myeloma, ovarian cancer, pancreatic cancer, prostate cancer and sarcoma but decreased in brain and CNS cancer (Fig. 1A). GEPIA website analysis showed that the expression of TMEFF1 mRNA was significantly increased in ovarian cancer, uterine corpus endometrial carcinoma, and uterine carcinosarcoma, but decreased in glioblastoma multiforme, acute myeloid leukemia, and thyroid carcinoma (Fig. 1B). The analysis of TMEFF1 in 174 endometrial cancer tissues and 91 normal endometrial tissues indicated that TMEFF1 mRNA was significantly highly expressed in endometrial cancer (Fig. 1C) $(P<0.01)$. Further analysis using the UALCAN website found that compared with patients with low/medium TMEFF1 expression (406 patients), the survival time of patients with high TMEFF1 expression (137 patients) with endometrial cancer was significantly shorter $(\mathrm{P}=0.025)$ (Fig. $1 \mathrm{D})$, suggesting that patients with endometrial cancer with high TMEFF1 expression have a poor prognosis.
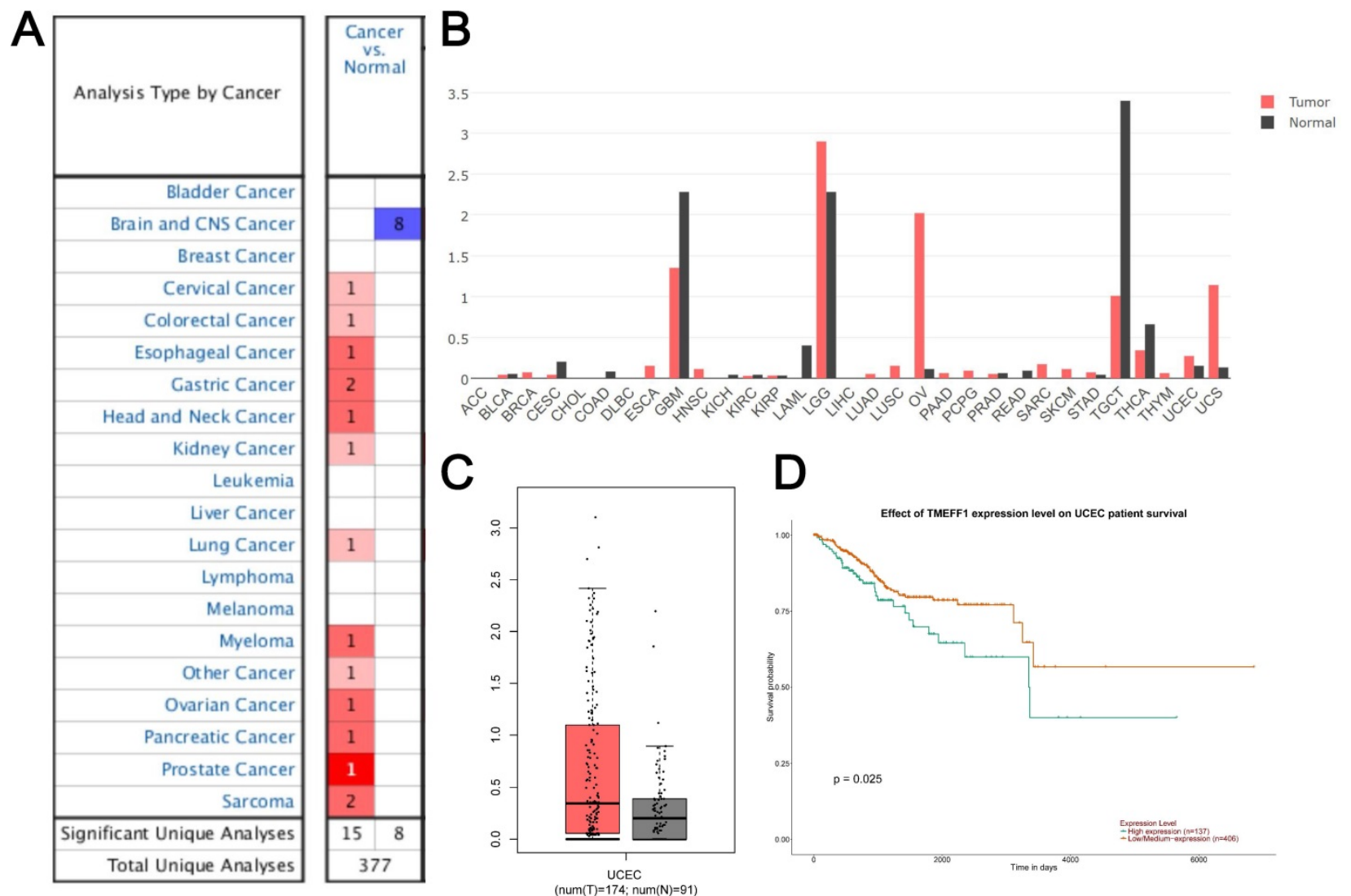

Figure 1. The expression of TMEFF 1 in the Oncomine dataset and GEPIA dataset of endometrial cancer patients. (A) TMEFF1 mRNA levels in different types of tumors in the Oncomine database. (B) TMEFF1 mRNA levels in different types of tumors in the GEPIA database. (C) TMEFF1 mRNA levels in endometrial cancer in the GEPIA database. (D) Correlation analysis of TMEFF1 expression with overall survival in UALCAN. Data are presented as the mean \pm SE. 


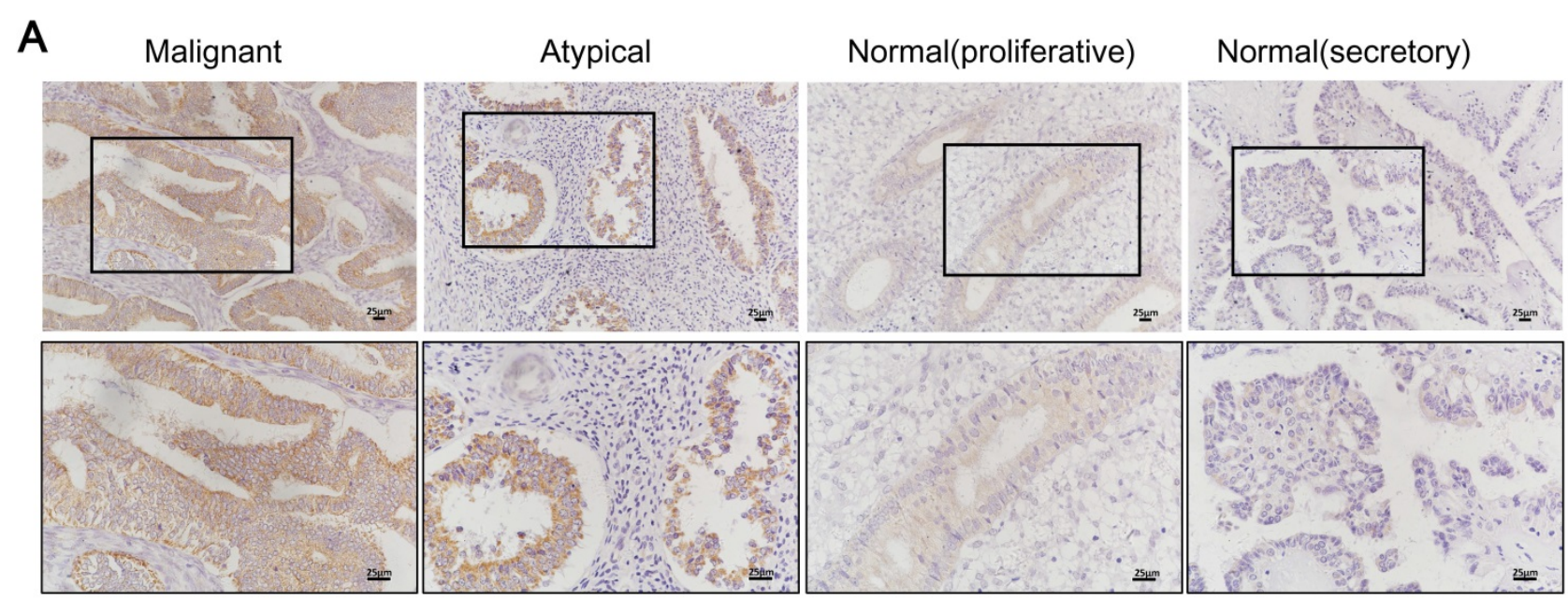

B

1

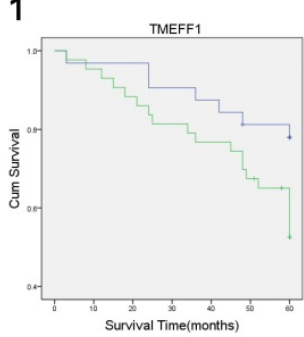

5

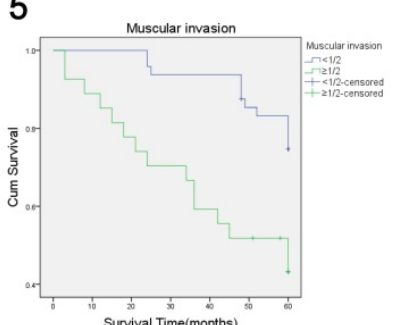

2

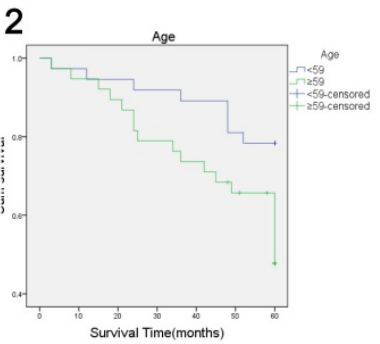

6

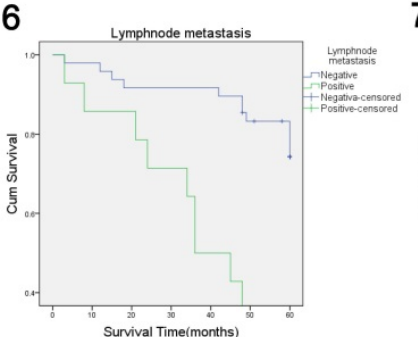

3
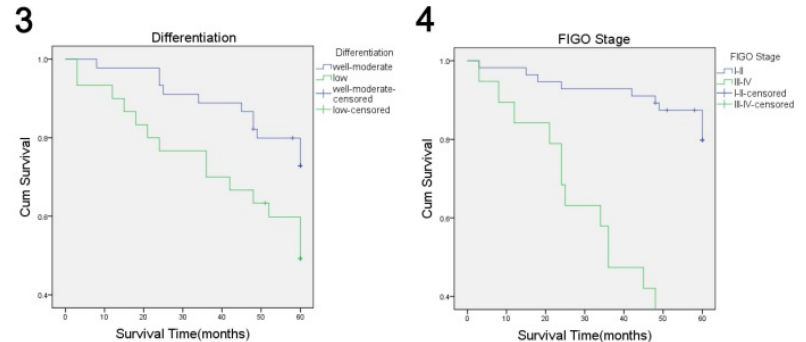

7

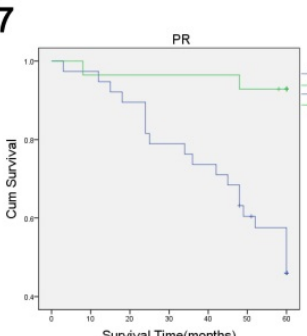

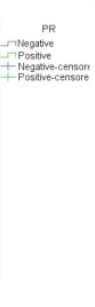

8

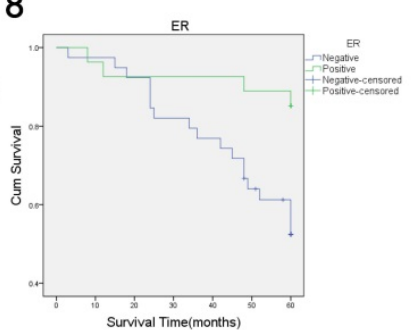

Figure 2. TMEFFI is significantly upregulated in various endometrial carcinomas and is significantly associated with patient prognosis. (A) Expression of TMEFF1 in various endometrial tissues $(\times 200 ; \times 400)$. (B) Analysis of factors related to endometrial prognosis. 1: TMEFF1 expression level; 2 : age at diagnosis; 3 : degree of differentiation; 4: FIGO stage; 5: depth of myometrial invasion; 6: lymphatic metastasis; g: PR(+); h: $\operatorname{ER}(+)$.

\section{TMEFFI expression in endometrial tissue}

TMEFF1 is highly expressed in endometrial carcinoma

TMEFF1 was mainly stained at the membrane, although staining was also evident in the cytoplasm (Fig. 2A). The positive expression rate of TMEFF1 in endometrial carcinoma was $82.67 \%$ (62 of 75 cases), which was significantly higher than that of atypical hyperplasia $(62.50 \%$ [15 of 24]) and normal endometrial tissue (58.33\% [21 of 36]) $(P=0.039$ and 0.006 , respectively). The high expression rate of TMEFF1 in endometrial carcinoma was $57.33 \%$ (43 of $75)$, which was significantly higher than that of normal endometrial tissue ( $25.00 \%$ [9 of 36]) $(P=0.001)$. The positive expression rates of TMEFF1 in severe, moderate, and mild endometrial atypical hyperplasia were $85.71 \%, 55.56 \%$, and $50.00 \%$, respectively, with the expression increasing with the severity of the lesion, although the difference was not statistically significant $(P>0.05)$. The positive rate of secretory endometrial tissue was $61.90 \%$ (13 of 21 ), that of proliferative endometrial tissue is $53.33 \%$ (8 of 15), and the difference was not statistically significant $(P>0.05)$ (Fig. 2A and Table 1).

Table 1. Expression of TMEFF1 in different endometrial tissues

\begin{tabular}{llllllll}
\hline Groups & Cases & Low & \multicolumn{3}{l}{ High } & $\begin{array}{l}\text { Positive } \\
\text { rate }(\%)\end{array}$ & $\begin{array}{l}\text { High expression } \\
\text { rate }(\%)\end{array}$ \\
\cline { 3 - 6 } & & $(-)$ & $(+)$ & $(++)$ & $(+++)$ & $82.67 \mathrm{ab}$ & $57.33 \mathrm{c}$ \\
Malignant & 75 & 13 & 19 & 27 & 16 & 82.50 & 45.83 \\
Atypical & 24 & 9 & 4 & 11 & 0 & 62.50 & 57.14 \\
Severe & 7 & 1 & 2 & 4 & 0 & 85.71 & 55.56 \\
Moderate & 9 & 4 & 0 & 5 & 0 & 55.56 & 25.00 \\
Mild & 8 & 4 & 2 & 2 & 0 & 50.00 & 25.00 \\
Normal & 36 & 15 & 12 & 8 & 1 & 58.33 & 20.00 \\
Proliferative & 15 & 7 & 5 & 3 & 0 & 53.33 & 28.57 \\
Secretory & 21 & 8 & 7 & 5 & 1 & 61.90 &
\end{tabular}

Note: The positive rate of TMEFF1 was significantly higher in the malignant group than in the atypical and normal groups $\left(P^{a}=0.039, P^{b}=0.006\right)$. The high expression rate of TMEFF1 was significantly higher in the malignant group than in the normal group $\left(P^{c}=0.001\right)$. 
Table 2. Relationship between TMEFF1 and clinical pathological parameters of endometrial carcinoma

\begin{tabular}{|c|c|c|c|c|c|c|c|c|c|}
\hline \multirow[t]{2}{*}{ Characteristics } & \multirow[t]{2}{*}{ Cases } & \multicolumn{2}{|c|}{ Low } & \multicolumn{2}{|l|}{ High } & \multirow[t]{2}{*}{ Positive rate $(\%)$} & \multirow[t]{2}{*}{$P$} & \multirow[t]{2}{*}{ High expression rate $(\%)$} & \multirow[t]{2}{*}{$P$} \\
\hline & & $(-)$ & $(+)$ & $(++)$ & $(+++)$ & & & & \\
\hline \multicolumn{10}{|c|}{ Age at diagnosis, years } \\
\hline$<59$ & 37 & 5 & 13 & 15 & 4 & 86.49 & \multirow[t]{2}{*}{$P>0.05$} & 51.35 & \multirow[t]{2}{*}{$\mathrm{P}>0.05$} \\
\hline$\geq 59$ & 38 & 8 & 6 & 12 & 12 & 78.95 & & 63.16 & \\
\hline \multicolumn{10}{|l|}{ FIGO stage } \\
\hline I & 50 & 12 & 13 & 18 & 7 & 76.00 & \multirow[t]{4}{*}{$P=0.021$} & 50.00 & \multirow[t]{4}{*}{$P=0.027$} \\
\hline II & 6 & 1 & 2 & 2 & 1 & 83.33 & & 50.00 & \\
\hline III & 16 & 0 & 3 & 6 & 7 & 100.00 & & 81.25 & \\
\hline IV & 3 & 0 & 1 & 1 & 1 & 100.00 & & 66.67 & \\
\hline \multicolumn{10}{|l|}{ Pathologic type } \\
\hline Endometrioid & 37 & 5 & 9 & 16 & 7 & 86.49 & \multirow[t]{4}{*}{$P>0.05$} & 62.16 & \multirow[t]{4}{*}{$P>0.05$} \\
\hline Serous & 23 & 5 & 6 & 7 & 5 & 78.26 & & 52.17 & \\
\hline Clear cell & 8 & 1 & 2 & 2 & 3 & 87.50 & & 62.50 & \\
\hline Others & 7 & 2 & 2 & 2 & 1 & 71.43 & & 42.86 & \\
\hline \multicolumn{10}{|l|}{ Differentiation } \\
\hline Well & 16 & 2 & 3 & 8 & 3 & 87.50 & \multirow[t]{3}{*}{$P>0.05$} & 68.75 & \multirow[t]{3}{*}{$P>0.05$} \\
\hline Moderate & 29 & 6 & 10 & 8 & 5 & 79.31 & & 44.83 & \\
\hline Poor & 30 & 5 & 6 & 11 & 8 & 83.33 & & 63.33 & \\
\hline \multicolumn{10}{|l|}{ ER } \\
\hline - & 39 & 6 & 11 & 10 & 12 & 84.62 & \multirow[t]{3}{*}{$P>0.05$} & 56.41 & \multirow[t]{3}{*}{$P>0.05$} \\
\hline+ & 27 & 5 & 7 & 14 & 1 & 81.48 & & 55.56 & \\
\hline Unknown & 9 & 2 & 1 & 3 & 3 & 77.78 & & 66.67 & \\
\hline \multicolumn{10}{|l|}{$\mathbf{P R}^{\mathbf{b}}$} \\
\hline - & 38 & 5 & 11 & 13 & 9 & 86.84 & \multirow[t]{3}{*}{$P>0.05$} & 57.89 & \multirow[t]{3}{*}{$P>0.05$} \\
\hline+ & 28 & 6 & 7 & 11 & 4 & 78.57 & & 53.57 & \\
\hline Unknown & 9 & 2 & 1 & 3 & 3 & 77.78 & & 66.67 & \\
\hline \multicolumn{10}{|c|}{ Muscular invasion } \\
\hline$<1 / 2$ & 48 & 9 & 13 & 17 & 9 & 81.25 & $P>0.05$ & 54.17 & $P>0.05$ \\
\hline$\geq 1 / 2$ & 27 & 4 & 6 & 10 & 7 & 85.19 & & 62.96 & \\
\hline LN metastasisc & & & & & & & & & \\
\hline- & 48 & 13 & 9 & 17 & 9 & 72.92 & $P=0.029$ & 54.17 & $P>0.05$ \\
\hline+ & 14 & 0 & 4 & 5 & 5 & 100.00 & & 71.43 & \\
\hline Unknown & 13 & 0 & 6 & 5 & 2 & 100.00 & & 53.85 & \\
\hline
\end{tabular}

a: Nine patients without ER detection. b: Nine patients without PR detection. c: Thirteen patients without lymphadenectomy.

\section{Relationship between TMEFF1 expression and clinicopathological parameters in endometrial carcinoma}

This study included 75 cases of endometrial carcinoma. The positive rate and high expression rate of TMEFF1 in early-stage cancer (I-II) were $76.78 \%$ (43 of 56 ) and $50.00 \%$ (28 of 56 ), respectively, which was significantly lower than those in the late stage (III-IV) (100.00\% [19 of 19] and 78.95\% [15 of 19]; $P=0.021$ and 0.027 , respectively). The positive expression rate of TMEFF1 in the positive lymph node metastasis group was $100 \%$ (14 of 14 ), which was significantly higher than that in the negative lymph node metastasis group (72.92\% [35 of 48]) $(P=0.029)$. The expression of TMEFF1 was not significantly associated with pathological type, degree of differentiation, $\mathrm{PR}(+)$ and $\mathrm{ER}(+)$ status, or depth of myometrial invasion (Table 2).

\section{Survival analysis and risk factors for prognosis of endometrial carcinoma}

Until November 2018, the patients' survival time ranged from 3 to 116 months. In total, 23 patients with endometrial carcinoma died due to cancer recurrence, 4 patients survived after cancer recurrence, 43 patients had cancer-free survival, 1 patient had non-recurrent death, and 4 patients were lost to follow-up. Univariate Kaplan-Meier analysis and log-rank test showed that high TMEFF1 expression was significantly associated with worse overall survival $(P=0.035)$. The high TMEFF1 expression group had a median survival time of 43 months, whereas the survival time in the low TMEFF1 expression group was 54 months. In addition, age at diagnosis $(<59$ years vs $\geq 59$ years), FIGO stage (I-II vs III-IV), type of differentiation (medium-high differentiation vs poor differentiation), depth of myometrial invasion $(<1 / 2$ vs $\geq 1 / 2$ ), lymph node metastasis ( $-\mathrm{vs}+$ ), and PR and ER status $(-$ vs +$)$ were also associated with poor prognosis $(P<0.05)$ (Fig. 2B and Table 3$)$.

We used Cox regression analysis to explore the relationships among different clinicopathological parameters, TMEFF1 expression, and prognosis. In addition to the age of diagnosis, FIGO stage, degree of differentiation, depth of myometrial invasion, lymph node metastasis and PR and ER status, high expression of TMEFF1 also affected the overall 
survival of patients with endometrial carcinoma and was an independent risk factor for prognosis. The higher expression of TMEFF1, the worse the prognosis $(P=0.044)$ (Table 4$)$.

Table 3. Univariate Kaplan-Meier prognosis analysis of endometrial carcinoma

\begin{tabular}{lll}
\hline Variable & Characteristics & (Log-rank) $P$-value \\
\hline Age at diagnosis & $<59$ years vs $\geq 59$ years & 0.011 \\
FIGO stage & I-II vs III-IV & $<0.001$ \\
Differentiation grade & Well-moderate vs poor & 0.026 \\
Muscular invasion & $<1 / 2$ vs $\geq 1 / 2$ & 0.001 \\
LN metastasis & Negative vs positive & $<0.001$ \\
PR & Negative vs positive & $<0.001$ \\
ER & Negative vs positive & 0.009 \\
TMEFF1 & Low vs high & 0.035 \\
\hline
\end{tabular}

Table 4. Univariate and multivariate Cox regression analysis of patients with endometrial carcinoma

\begin{tabular}{lllll}
\hline Variables & \multicolumn{2}{l}{ Univariate analysis } & \multicolumn{2}{l}{ Multivariate analysis } \\
\cline { 2 - 5 } & $P$-value & $\begin{array}{l}\text { Hazard ratio } \\
(95 \% \mathrm{CI})\end{array}$ & $P$-value & $\begin{array}{l}\text { Hazard ratio } \\
(95 \% \mathrm{CI})\end{array}$ \\
\hline $\begin{array}{l}\text { Age at diagnosis } \\
\text { FIGO stage }\end{array}$ & 0.017 & $2.743(1.199-6.273)$ & 0.929 & $0.942(0.253-3.503)$ \\
& $<0.001$ & $7.640(3.488-16.732)$ & 0.004 & $\begin{array}{l}27.107 \\
(2.795-262.904)\end{array}$ \\
$\begin{array}{l}\text { Differentiation } \\
\text { grade }\end{array}$ & 0.033 & $2.281(1.067-4.877)$ & 0.323 & $1.748(0.578-5.288)$ \\
Muscular invasion & 0.002 & $3.276(1.528-7.025)$ & 0.025 & $4.948(1.223-20.026)$ \\
LN metastasis & $<0.001$ & $5.183(2.255-11.911)$ & 0.213 & $0.234(0.024-2.308)$ \\
PR & 0.002 & $0.104(0.024-0.445)$ & 0.314 & $0.314(0.043-2.285)$ \\
ER & 0.016 & $0.262(0.088-0.776)$ & 0.18 & $0.398(0.103-1.533)$ \\
TMEFF1 & 0.044 & $2.421(1.023-5.728)$ & 0.302 & $3.037(0.368-25.068)$ \\
\hline
\end{tabular}

A
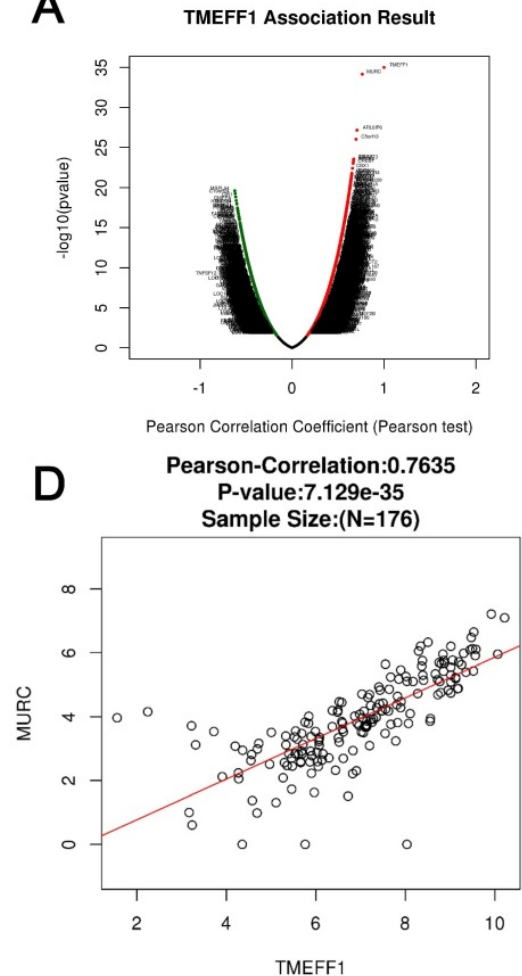

B
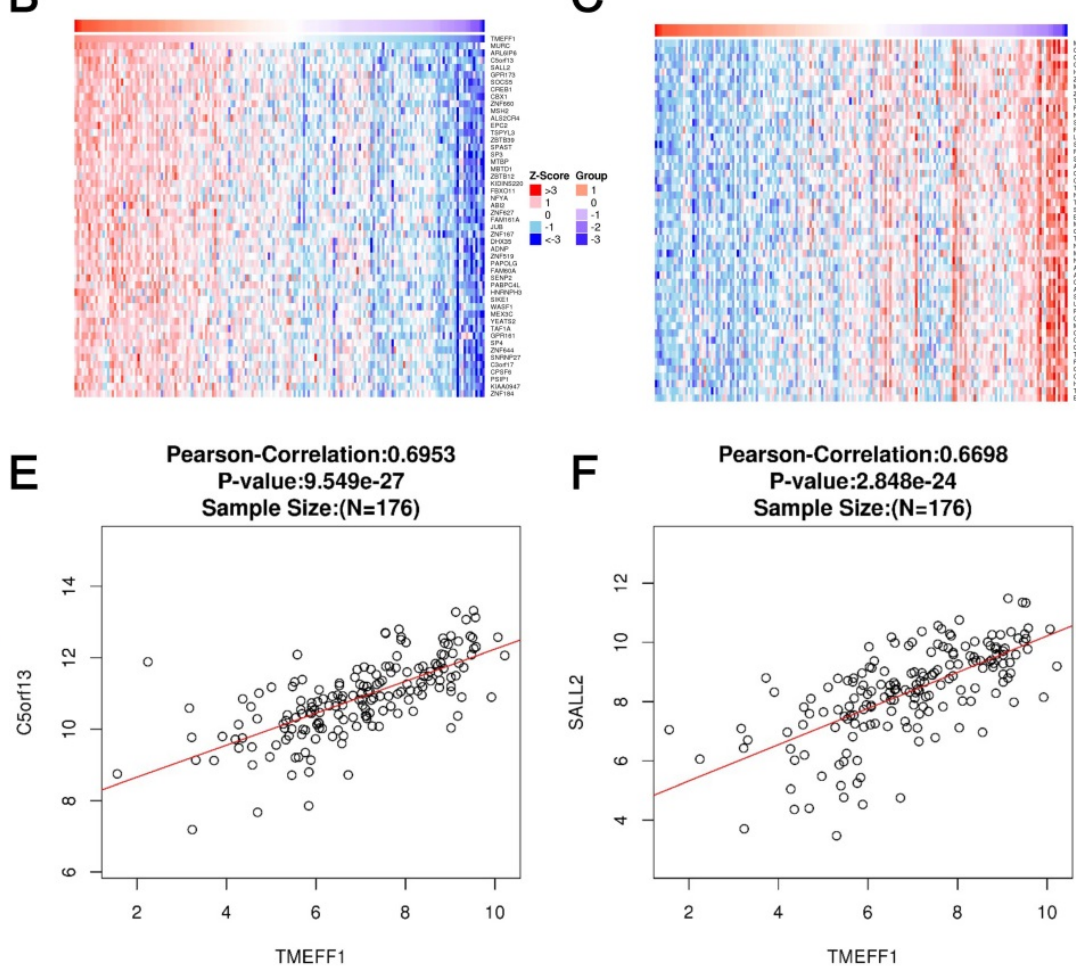

Figure 3. Differentially expressed genes correlated with TMEFFI in endometrial carcinoma. (A) Pearson's test was used to analyze the correlation between TMEFF1 and differentially expressed genes in UCEC. (B and C) Genes positively correlated and negatively correlated with TMEFF1 in UCEC, as shown in heatmaps (top 50). Red indicates positively correlated genes, and green indicates negatively correlated genes. (D-F) Correlation of TMEFFI expression with the expression of MURC (D), C5orf13 (E) and SALL23 (F) according to Pearson's test, as shown in scatter plots. 
A

Cellular component

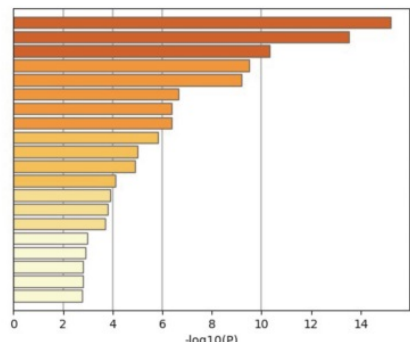

C

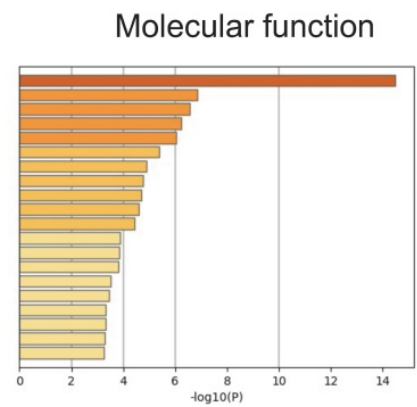

E

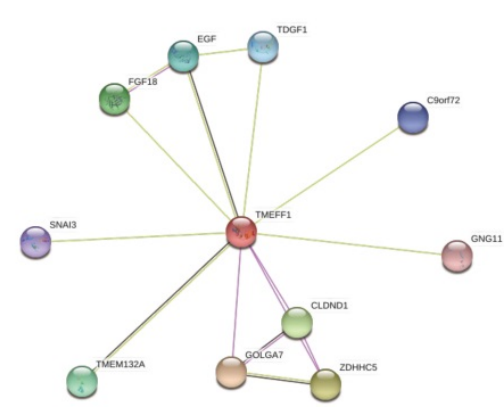

B
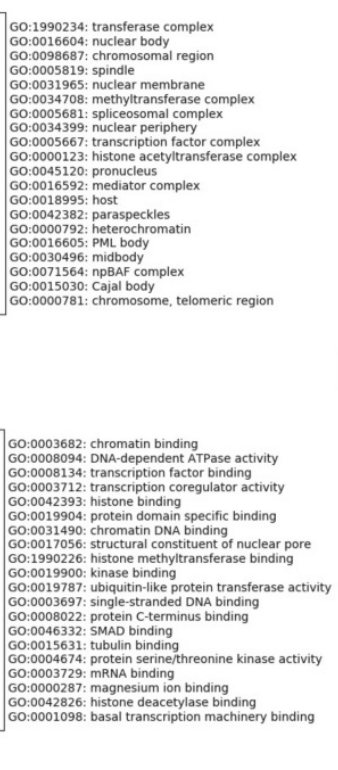

D

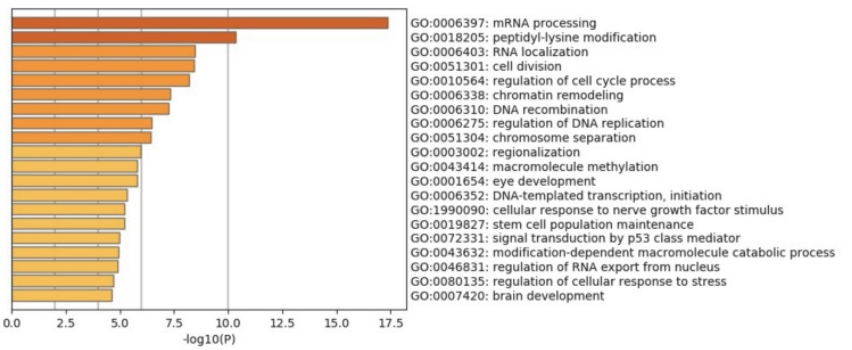

KEGG pathway

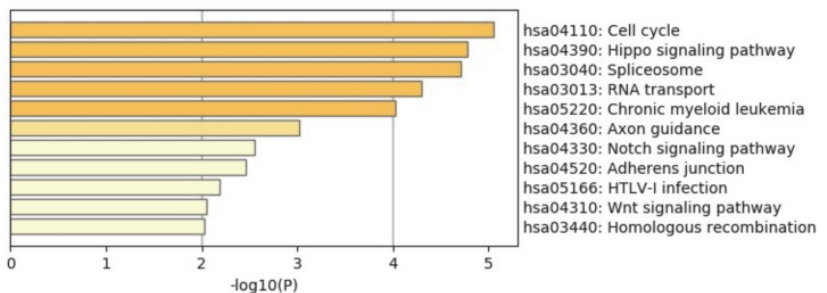

F

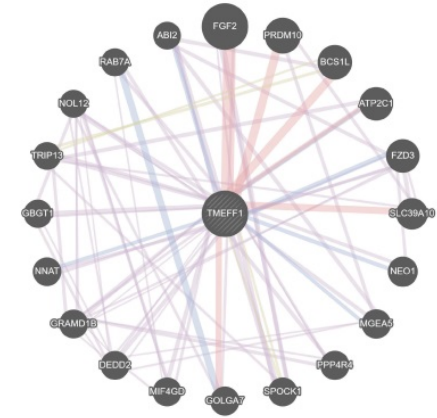

Figure 4. The significantly enriched GO annotation and KEGG pathway terms of genes coexpressed with TMEFFI in UCEC were analyzed with Metascape, and proteins interacting with TMEFFI were analyzed in the STRING database and GeneMANIA database. (A) Top 20 enriched with cellular component terms for the TMEFF1-related genes, as shown in a bar graph colored by $P$-values. (B) Top 20 enriched biological process terms for the TMEFF1 co-expressed genes, as shown in a bar graph colored by $P$-values. (C) Top 20 enriched molecular function terms for to the TMEFF1 co-expressed genes, as shown in a bar graph colored by $P$-value. (D) KEGG enriched terms colored by P-value. (E) PPI analysis using the STRING database. (F) PPI analysis using the GeneMANIA database.

GO and KEGG enrichment analyses were performed to determine the functions of TMEFF1 and related differentially expressed genes. The GO results showed that TMEFF1 and its associated genes are mainly located in transferase complexes, the nuclear body, the chromosomal region, the spindle, the nuclear membrane, the nuclear periphery, and chromosomes (Fig. 4A and Table S1). In addition, they are mainly involved in the cell cycle, the regulation of mitosis, epigenetics, neurodevelopment, cell biological signal transduction and other biological processes, such as mRNA processing, peptidyl-lysine modification, cell division, regulation of the cell cycle, chromatin remodeling, cellular response to nerve growth factor stimulus, brain development, signal transduction by p53 class mediators, etc. (Fig. 4B and Table S2). The molecular functions of TMEFF1 and its associated genes were found to mainly include regulating the activities of DNA-dependent ATPases, transcription coregulators, ubiquitin-like protein transferases, and protein serine/threonine kinases, and TMEFF1 and its associated proteins were suggested to be able to bind to chromatin, transcription factors, histones, protein domains, chromatin DNA, single-stranded DNA, protein C termini, tubulin, etc.; they can also interact with histone methyltransferases, kinases, histone deacetylase, etc. (Fig. 4C and Table S3).

The KEGG enrichment analysis results showed that the signaling pathways involving TMEFF1 and its associated genes include the cell cycle, Hippo signaling, spliceosome, RNA transport, and chronic myeloid leukemia pathways. (Fig. 4D and Table S4).

\section{TMEFF 1-related kinase, miRNA or transcription factor target network in endometrial cancer}

To further explore the functional targets of TMEFF1 in endometrial cancer, we analyzed the kinases, miRNAs and transcription factors that could serve as targets of genes positively related to TMEFF1 
by GSEA. The top 5 identified kinases were ATM serine/threonine kinases, TTK protein kinases, ATR serine/threonine kinases, polo-like kinase 1, and cyclin-dependent kinase 2 , which are mainly related to DNA damage, mitosis and the cell cycle. The miRNA target network included MIR-381, MIR-154, MIR-487, MIR-30A-3P, MIR-30E-3P, MIR-369-3P, MIR-212, and MIR-132. The transcription factor target network included proteins mainly related to DNA replication and repair and cell cycle regulation, including V\$E2F1_Q6, V\$E2F_02, V\$E2F1DP1_01, V\$E2F1DP2_01, and V\$E2F4DP2_01 (Table 5 and Table S5-S7).

Table 5. The Kinase, miRNA and transcription factor-target networks of TMEFF1 in Uterine Corpus Endometrial Carcinoma

\begin{tabular}{llll}
\hline $\begin{array}{l}\text { Enriched } \\
\text { Category }\end{array}$ & Geneset & LeadingEdgeNum & FDR \\
\hline Kinase Target & ATM serine/threonine kinase & 63 & 0.002129 \\
& TTK protein kinase & 7 & 0.055353 \\
& ATR serine/threonine kinase & 13 & 0.055353 \\
& polo like kinase1 & 11 & 0.0599611 \\
& cyclin dependent kinase 2 & 3 & 0.18948 \\
miRNA Target & CTTGTAT,MIR-381 & 110 & 0 \\
& GTATGAT,MIR-154,MIR-487 & 37 & 0 \\
& ACTGAAA,MIR-30A-3P, & 89 & 0 \\
& MIR-30E-3P & 110 & 0 \\
& GTATTAT,MIR-369-3P & 68 & 0 \\
Transcription & GACTGTT,MIR-212,MIR-132 & 94 & 0 \\
Factor Target & V\$E2F1_Q6 & 93 & 0 \\
& V\$E2F_02 & 92 & 0 \\
& V\$E2F1DP1_01 & 92 & 0 \\
\hline
\end{tabular}

\section{PPI analysis using the STRING database and GeneMANIA database}

To better understand the relationship between TMEFF1 and UCEC, we used STRING and GeneMANIA to perform protein-protein interaction (PPI) enrichment analysis (Fig. 4E and F). The results show that proteins that interact with TMEFF1 are involved in cell proliferation, differentiation, migration, apoptosis, autophagy, cell adhesion, protein transport, calcium transport, and various signal transduction processes and promote the development of nerves and embryos.

\section{Genomic changes in TMEFFI in endometrial cancer}

Based on the sequencing data of UCEC patients in the TCGA database, we used cBioPortal to determine the type and frequency of TMEFF1 changes in UCEC. TMEFF1 was altered in 29 of the $1638(2 \%)$ UCEC patients (Fig. 5A). These alterations included the following: amplification (3 patients; $0.2 \%$ ), deep deletion (6 patients; $0.4 \%$ ), truncating mutation of unknown significance (4 patients; $0.2 \%$ ) and missense mutation of unknown significance (16 patients; $1 \%$ ). These results show that the TMEFF1 gene mutation has a very low incidence in endometrial cancer. And TMEFF1 gene mutation had no significant effect on Overall Survival (OS), Disease Free Survival(DFS), Progression Free Survival (PFS) or Disease Specific Survival(DSS) in patients with endometrial cancer $(P>0.05)$ (Fig. 5B-E).

\section{Establishment of a low TMEFF 1 expression endometrial carcinoma cell line}

We first compared the expression levels of TMEFF1 in different endometrial carcinoma cell lines (Ishikawa, HEC-1-B, and HEC-1-A) and then selected Ishikawa and HEC-1-B cell lines with high TMEFF1 expression for knockdown of the TMEFF1 gene. The interference efficiency was verified by real-time PCR and western blot experiments, with the expression of TMEFF1 gene and protein significantly lower in the interference group than in the control group (Fig. 6A-C).
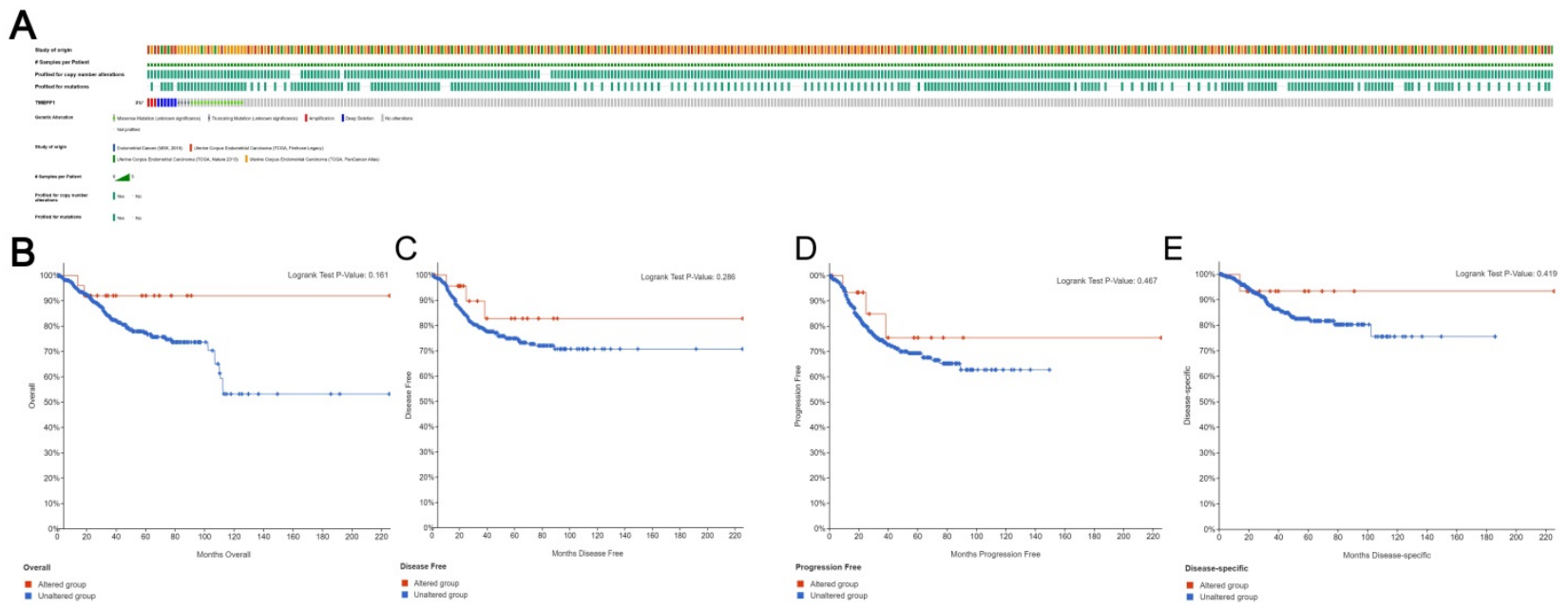

Figure 5. TMEFFI gene mutations and their effect on the survival and prognosis of endometrial carcinoma patients. (A)Mutations of the TMEFF1 gene in the cBioPortal database. (B-E) Effect of the mutations of the TMEFF1 gene on the OS (B), DFS (C), PFS (D), and DSS (E) of endometrial carcinoma patients. 


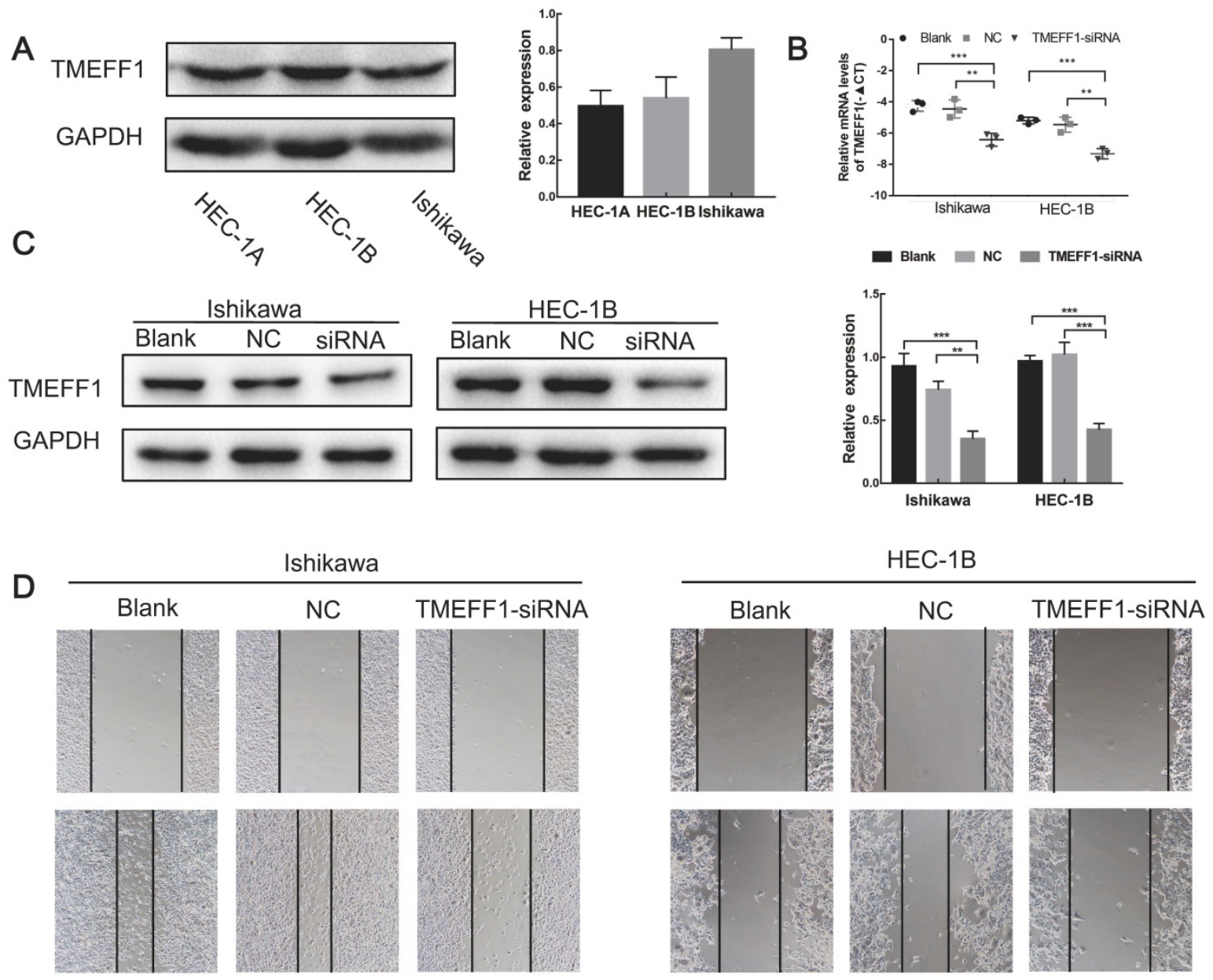

E

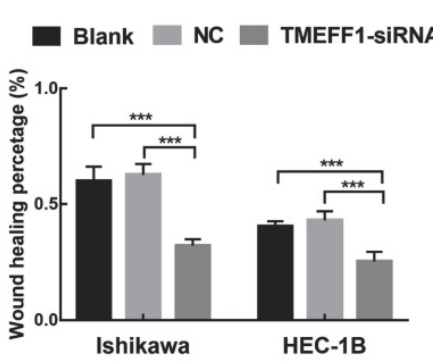

G

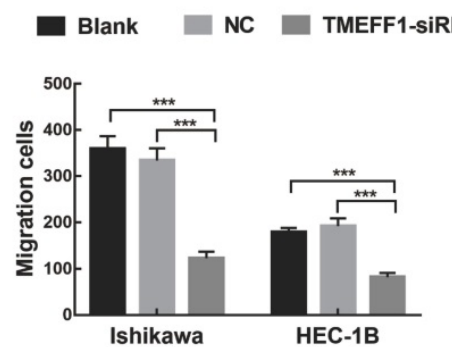

ISK

ISK-NC

ISK-siRNA

$\mathrm{HB}$

HB-NC

HB-siRNA
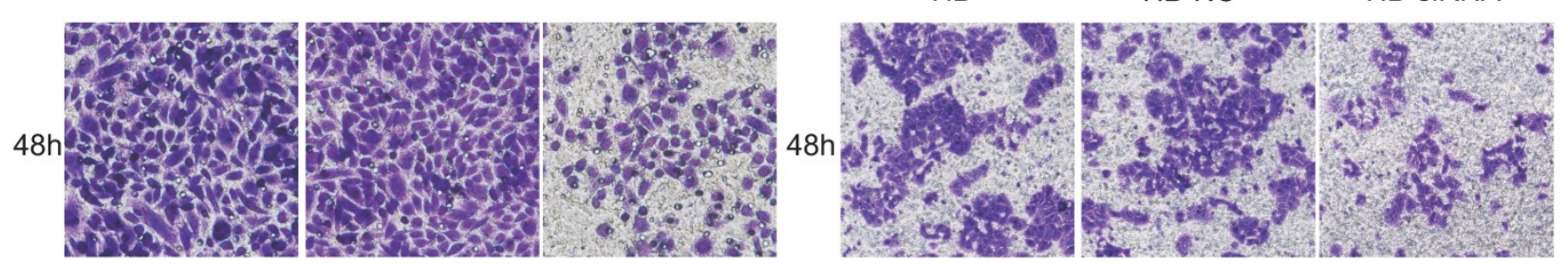

Figure 6. Knockdown of TMEFF1 inhibits the migration and invasion of Ishikawa and HEC-1B cells. (A) Expression of TMEFF1 in endometrial carcinoma cell lines. TMEFF1 (Molecular Weight): $41 \mathrm{kDa}$; GAPDH (Molecular Weight): 36kDa. (B and C) After TMEFF1 knockdown in the intimal cancer cell lines Ishikawa and HEC-1B, the expression of TMEFF1 mRNA and protein was determined. (D and E) Scratch test to determine the effect of TMEFF1 expression on the migration of Ishikawa and HEC-1B cells. $(\mathbf{F}$ and $\mathbf{G})$ Transwell assay assessing the effect of TMEFF1 expression on the invasion of Ishikawa and HEC-1B cells. Data are presented as the mean \pm SEM $(\mathrm{n}=3$ per group). $* * P$ $<0.01$ and $* * * P<0.001$.

TMEFFI knockdown inhibits the migration of endometrial carcinoma cells

Cell scratch experiments showed that the migration ability of Ishikawa and HEC-1-B cells decreased after knockdown of the TMEFF1 gene (Fig. $6 \mathrm{D}$ and E), indicating that TMEFF1 promotes the migration of endometrial carcinoma cells. 


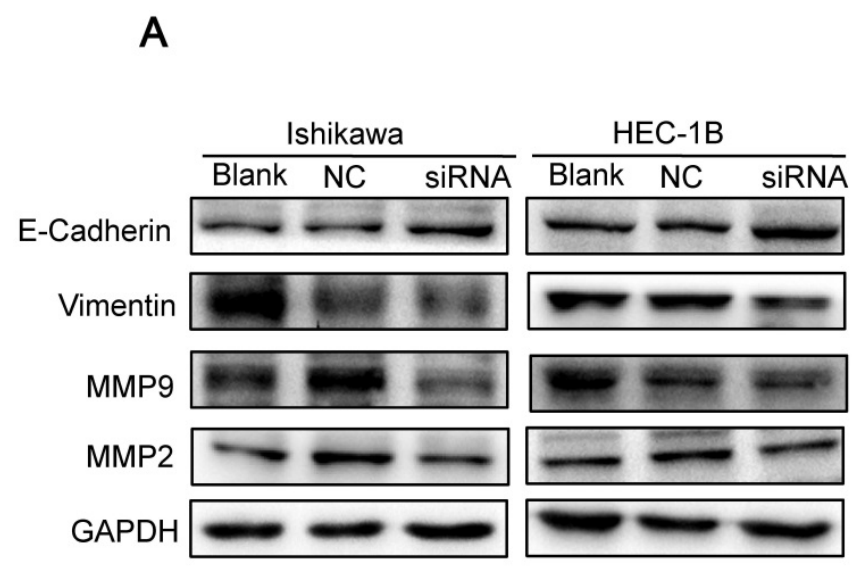

B
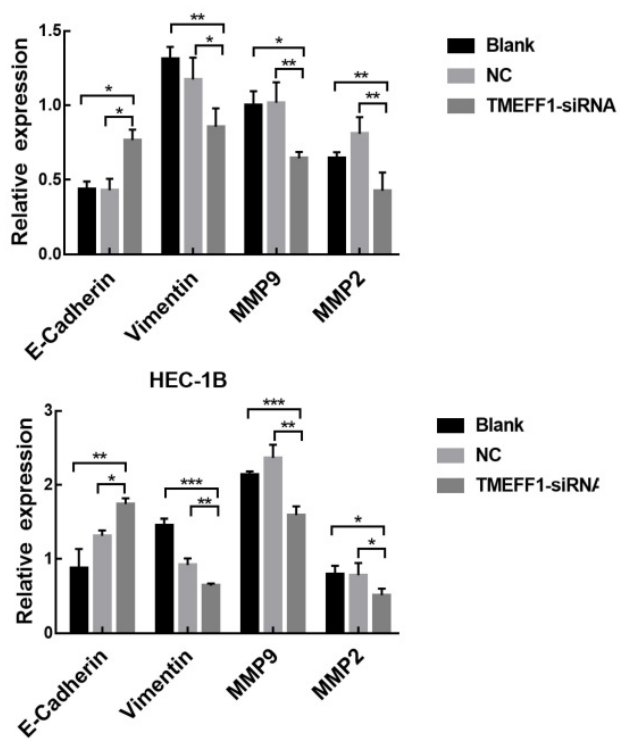

Blank

NC

TMEFF1-siRN $/$

Figure 7. TMEFF1 is involved in the promotion of epithelial-mesenchymal transition in Ishikawa and HEC-1B cells. (A and B) Western blot analysis indicating that TMEFF1 increases the expression of vimentin, MMP2, and MMP9 in Ishikawa and HEC-1B cells and decreases the expression of E-cadherin. Data are presented as the mean \pm SEM ( $\mathrm{n}=3$ per group). $* P<0.05, * * P<0.01$, and $* * * P<0.001$.

\section{TMEFFI knockdown inhibits the invasion of endometrial carcinoma cells}

Transwell assays revealed that TMEFF1 knockdown in Ishikawa and HEC-1-B cell lines significantly reduced cell invasiveness (Fig. 6F and G), indicating that TMEFF1 promotes the invasion of endometrial carcinoma cells.

\section{TMEFFI affects key proteins involved in the regulation of EMT}

EMT plays an important role in the early stages of the metastatic spread of cancer by inducing cell movement and allowing cells to acquire invasive potential. To further explore the mechanism underlying the effect of TMEFF1 on the invasion and migration of endometrial carcinoma cells, we used western blot to determine the changes in the key EMT proteins E-cadherin, Vimentin, MMP2, and MMP9 between before and after TMEFF1 knockdown. The results showed that Vimentin, MMP2, and MMP9 expression levels were decreased and E-cadherin increased after inhibition of the expression of the TMEFF1 gene (Fig. 7A and B). Thus, TMEFF1 is involved in the regulation of EMT and affects the malignant behavior of endometrial carcinoma cells.

\section{TMEFF 1 activates PI3K of the AKT and MAPK signaling pathways}

To further explore the mechanism through which TMEFF1 affects the malignant behavior of endometrial carcinoma cells, we examined the pathway node proteins and found that, compared with the control group, knockdown of the TMEFF1 gene decreased the p-MEK/MEK, p-ERK/ERK, p-PI3K/PI3K, and p-AKT/AKT ratios (Fig. 8A and B). These results indicate that TMEFF1 activates the MAPK and PI3K/AKT signaling pathways in endometrial carcinoma.

\section{The transcription factor p53 in endometrial cancer cell lines cannot directly regulate the expression of TMEFF 1}

TP53 gene mutations are more abundant in endometrial cancer tissue than normal endometrial tissue. TP53 mutations are closely related to tumors. Further analysis using the UALCAN website found that the expression level of TMEFF1 mRNA in the TP53-mutant group was higher than that in the normal control group and TP53-unmutated group $(P$ $<0.05, P<0.001$ ) (Fig. 9A), indicates that TMEFF1 may play an important role in UCEC with TP53 mutations. Further exploration of the regulatory effect of the transcription factor p53 on TMEFF1 was carried out via ChIP experiments, but it was found that p53 cannot bind to the promoter region of TMEFF1 in endometrial cancer cells $(P>0.05)$ (Fig. 9B and $C)$.

\section{Discussion}

The vast majority of endometrial carcinoma occurs in postmenopausal women. However, the age of incidence is becoming younger. About 25\% of endometrial carcinoma cases are advanced when diagnosed. These patients have an unsatisfactory objective response rate to chemotherapy and endocrine therapy and poor prognosis. However, the molecular mechanisms of endometrial carcinoma 
development, progression, and invasion/migration have not been clearly identified [20-22]. The TMEFF1 gene was originally discovered as a gene encoding the secretory protein of the pituitary gland of Xenopus laevis, called X7365, also known as tomoregulin-1 [23]. Early research into TMEFF1 focused on regulation of the central nervous system and embryonic development [24]. TMEFF1 was found to be associated with neurological diseases, including Parkinson's disease and GM2 gangliosidosis [25, 26]. Subsequently, TMEFF1 was confirmed to be a member of the CTA family [7]. The CTA family is involved in the occurrence and development of cancers and is currently a research topic of interest in cancer immunodiagnosis and immunotherapy [4,5]. The expression of the CTA family XAGE- $1 b$ is significantly higher in liver cancer tissues and peripheral blood than in a non-liver cancer group. Because the expression level of XAGE-1b is closely associated with the 1-year recurrence rate of patients [27], it can be useful for early diagnosis and prognosis. The expression levels of the CTA family members MAGE-3 and MAGE-4 mRNA are significantly higher in the peripheral blood of metastatic hepatocellular carcinoma patients than in a non-metastasis group, and they can be used to determine whether hepatoma cells undergo blood vessel metastasis [28].

As a new member of the CTA family, the function of TMEFF1 is not yet clear. In this study, we sought to explore the role of TMEFF1 in endometrial carcinoma and its mechanisms. We found that
TMEFF1 is highly expressed in endometrial carcinoma tissues and promotes the invasion and migration of endometrial carcinoma cells. However, it is worth noting that the early research into TMEFF1 in cancer indicated that it acts as a cancer suppressor, highly expressed in normal human brain tissues and lowly expressed in brain malignant cancers. In addition, in four kinds of brain cancer cells, TMEFF1 exhibited medium and low expression, with expression of TMEFF1 in brain cancers causing growth inhibition [9]. However, high expression of TMEFF1 was also detected in various cancer cell lines such as prostate cancer, ovarian cancer, and pancreatic cancer, but its function is not known $[8,29]$.

Through the Oncomine database and GEPIA online analysis tool TMEFF1 was found to be significantly highly expressed in esophageal cancer, gastric cancer, ovarian cancer, pancreatic cancer, prostate cancer and uterine corpus endometrial carcinoma. To further explore the relationship between the expression of TMEFF1 in endometrial cancer and the prognosis, Kaplan-Meier survival analysis via UALCAN showed that the overall survival (OS) of patients with high expression of TMEFF1 was significantly worse than that of patients with low expression, indicating that high expression of TMEFF1 can indicate a poor prognosis of patients with endometrial cancer and that TMEFF1 expression has potential as a molecular marker for clinical diagnosis and prognostic evaluation.

B
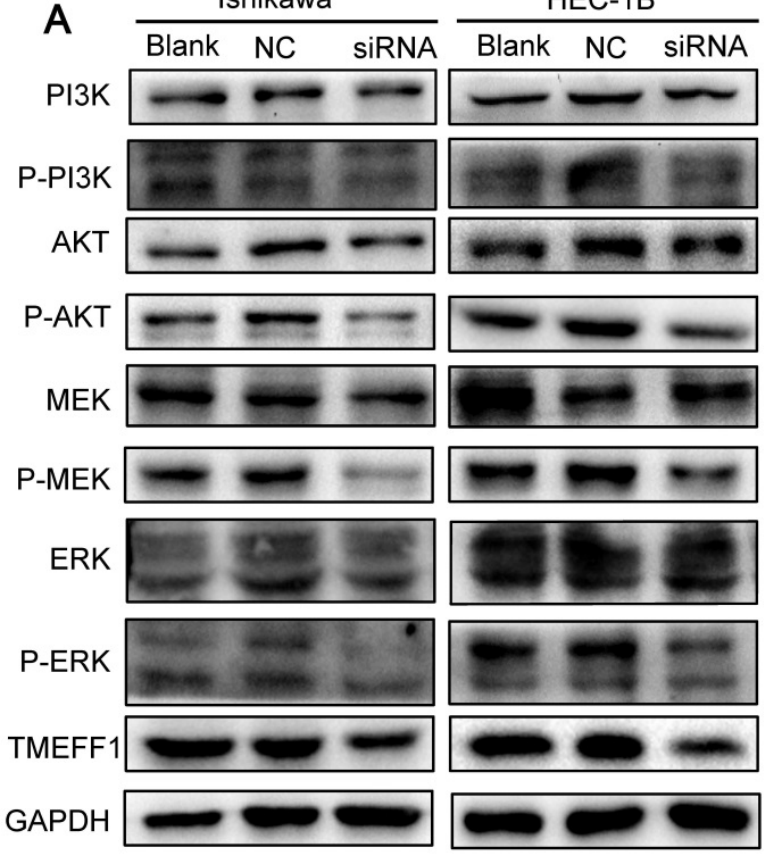
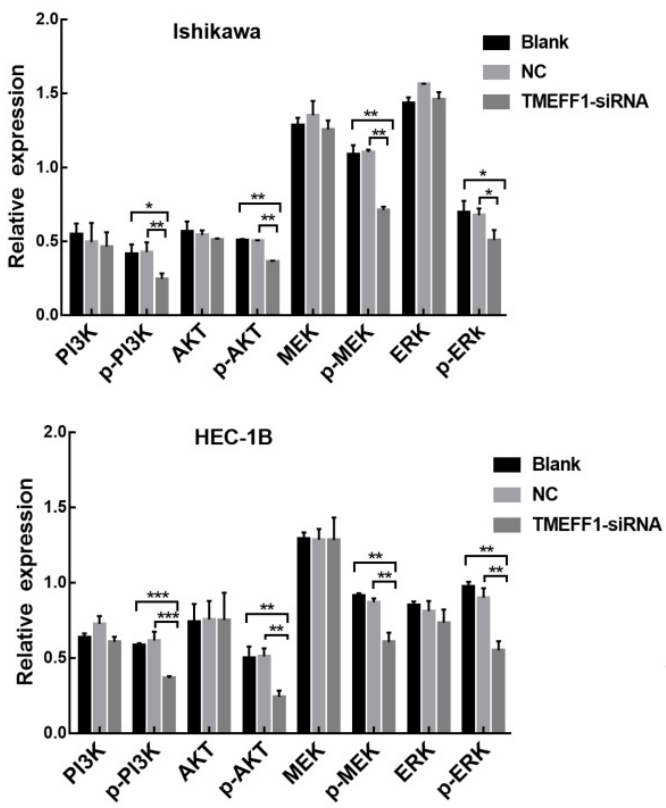

Figure 8. TMEFFI activates the MAPK and PI3K/AKT signal transduction pathways in Ishikawa and HEC-IB cells. (A and B) Western blot was used to determine the phosphorylation of MAPK pathway node proteins and PI3K/AKT pathway node proteins in Ishikawa and HEC-IB cell lines before and after TMEFFI transfection. Data are presented as the mean \pm SEM $(\mathrm{n}=3$ per group). $* P<0.05$, $* * P<0.01$, and $* * * P<0.001$. 
A

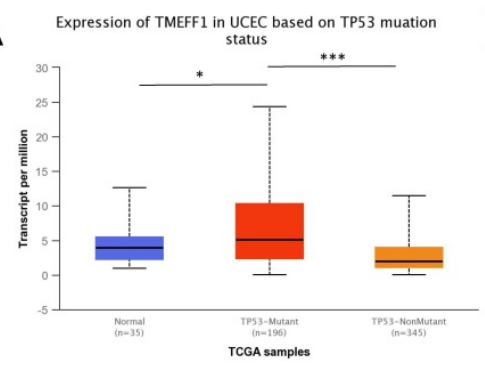

B

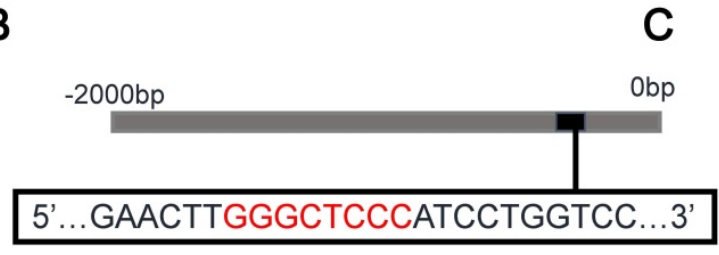

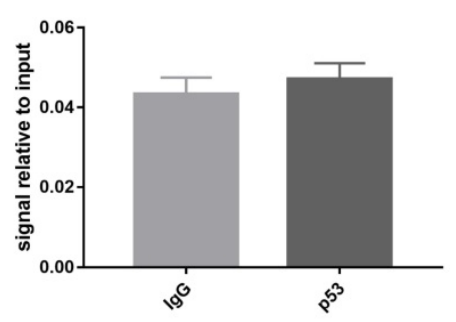

Figure 9. TMEFF1 is not regulated by p53 in endometrial carcinoma cells. (A) Relative expression of TMEFF1 in normal individuals and UCEC patients with different TP53 mutation statuses. (B)Predicted p53 binding sites in the promoter region of TMEFF1. (C) The promoter region of TMEFF1 did not bind with the transcription factor p53 in Ishikawa cells. Data are presented as the mean \pm SEM ( $n=3$ per group).

Previous studies have found that TMEFF1 is highly expressed in ovarian cancer and is an independent risk factor for prognosis. TMEFF1 promotes the malignant behavior of ovarian cancer by activating the PI3K/AKT and MAPK pathways [10]. TMEFF1 plays a distinctly different role in cancerogenesis in different cancer tissues. To further verify the expression of TMEFF1 in endometrial cancer tissues and analyze its relationship with clinicopathological parameters and prognosis, in this study, the expression of TMEFF1 was determined in 135 endometrial tissue samples by immunohistochemistry. Our results showed that TMEFF1 was highly expressed in endometrial carcinoma, that its expression was closely associated with FIGO stage and lymph node metastasis, and that it was an independent predictor of survival. The results also showed that the high expression rate of TMEFF1 in the atypical endometrial hyperplasia group was significantly higher than that in the normal endometrium group, and lower than that in the malignant group, which demonstrates that the expression of TMEFF1 was related to the malignant progression of endometrial cancer, and can monitor the malignant transformation and prognosis in patients with atypical endometrial hyperplasia. The above data demonstrate that TMEFF1 has malignant potential in human endometrial carcinoma.

More than $90 \%$ of solid cancer deaths are largely due to metastasis [30]. Cancer metastasis is a complex process that includes invasion, neovascularization and intravascular penetration, survival and antianoikis in the blood circulation, and extravasation and colonization [30-33]. However, the mechanisms of the above process remain unclear. In the case of independent expression, TMEFF1 was preferentially expressed in the cell membrane. However, after the binding of TMEFF1 to addicsin, which is a new protein in the amygdala of mice, the distribution of TMEFF1 in the cell membrane was inhibited so that TMEFF1 mainly redistributed to the endoplasmic reticulum and suppressed the migration of cells [34]. This indicates that TMEFF1 participates in the migration process of cells. In this study, the results of cell scratch and Transwell assays showed that TMEFF1 knockdown inhibits cell invasion and migration in Ishikawa and HEC-1B cells. These results support the hypothesis that TMEFF1 is a cancer-promoting gene in endometrial carcinoma. The EMT process is considered to be a key mechanism of metastatic changes. Many CTA family members (e.g., SSX, MAGE-D4B, CAGE, piwil2, and CT45A1) are involved in the regulation of cancer cell EMT, upregulating EMT and metastatic genes and promoting EMT and cancer metastasis [35]. We found that TMEFF1 inhibited the expression of E-cadherin and upregulated the mesenchymal marker vimentin and the metalloproteinases MMP2 and MMP9. These results indicate that TMEFF1 is involved in promoting EMT. Studies have shown that when the EMT transcriptional factors Snail, Slug, and E47 are upregulated, TMEFF1 expression is elevated [36]. These transcription factors are significant inducers of EMT and strongly inhibit the expression of E-cadherin $[36,37]$, indicating that TMEFF1 is the intermediate link between these transcription factors affecting E-cadherin.

Mutation in the tumor suppressor gene TP53 is associated with aggressive and advanced tumors [38]. It is more common in high-grade, poorly differentiated, and progesterone receptor-negative cases of endometrial cancer than in low-risk cases [39]. The prognosis of endometrial cancer patients with TP53 mutation is worse than that of patients without mutation [39]. We found that in endometrial cancer, the expression level of TMEFF1 mRNA in the TP53-mutant group was higher than that in the normal endometrial group and the TP53-unmutated group. This is consistent with the previous discovery that TMEFF1 is highly expressed in TP53-mutant breast cancer [40], indicating that TMEFF1 plays a greater role in TP53-mutant tumors. We previously confirmed that the expression of TMEFF1 in ovarian cancer is regulated by the transcription factor p53 (mutant)[10], but the same regulatory effect has not been found in endometrial cancer, indicating that 
there may be other links between TMEFF1 and TP53 in endometrial cancer, which need to be further explored.

Through the cBioPortal database, it was found that TMEFF1 is genetically altered in endometrial cancer, with alterations mainly including amplifications, deep deletions, truncating mutations and missense mutations. However, these mutations had no significant effect on OS, DFS, PFS or DSS in patients with endometrial cancer. To better clarify the molecular mechanism, we further constructed a gene co-expression network with TMEFF1 and their top 50 neighbor genes, suggesting that TMEFF1 may exert their function by interacting with proteins. Studies have found that the TMEFF1 domain EGF can interact with the CUB domain of ST14, and such an interaction activates ST14 [29], which play a role in the degradation of extracellular matrix and the promotion of tumor growth, infiltration, metastasis and other malignant processes [41]. This indicates that TMEFF1 can participate in tumor progression through its interacting proteins. GO enrichment analysis showed that TMEFF1 and its related genes are mainly located in the transferase complex, nuclear body, chromosomal region and other regions, combined with chromatin, transcription factor, histone, etc., and can regulate the activity of DNA-dependent ATPase, transcription coregulator, ubiquitin-like protein transferase and other enzymes, indicating that TMEFF1 and its related genes are mainly involved in gene epigenetics and cell biological signal transduction. Many studies have shown that TMEFF1 can regulate multiple molecular mechanisms and related signal pathways. TMEFF1 prevents the binding of Cripto- 1 to the ALK4 receptor by binding to the CFC domain of Cripto-1, thereby inhibiting nodal signaling [42, 43]; TMEFF1 acts as a downstream signaling factor of TGF- $\beta$ R2 and acts directly on Smad2/3, inhibiting the BMP signal to reduce the threshold required for the growth inhibitory signal of hair follicle stem cells, thereby promoting hair follicle stem cell self-renewal [44]. TMEFF1 regulates the development of cardiac hypertrophy in TAC-induced cardiac hypertrophy by inhibiting TGF $\beta$ noncanonical (TAK1-JNK) pathways in the myocardium [45].

KEGG enrichment analysis revealed significant enrichment in the cell cycle, Hippo signaling, spliceosome, RNA transport and other pathways. The above signaling pathways can participate in the occurrence and development of a variety of tumors and are closely related to the occurrence and development of UCEC [46-49]. This study found that TMEFF1 promotes the malignant behavior of endometrial carcinoma cells by activating the MAPK and PI3K/AKT signaling pathways, which is consistent with previous findings in ovarian cancer, suggesting that this generality may be related to the EGF-like domain of TMEFF1 [10]. TMEFF1 is a member of the EGF family because its extracellular region contains a modified EGF-like domain that is highly homologous to the growth factors of the EGF family. The EGF family is closely associated with the occurrence and development of cancers. When EGFR binds to the ligands, the receptors autophosphorylate and transmit information along various downstream signaling pathways such as PI3K/AKT and MAPK to promote cell proliferation and increase cancer cell migration and invasion $[50,51]$. The EGF-like domain of TMEFF1 can phosphorylate tyrosine of the erbB4 receptor $[23,24,52]$, which in turn may activate the MAPK and PI3K/AKT pathways to promote the malignant behavior of cancers.

Targeted drugs for inhibiting the MAPK or PI3K pathway have been used clinically but, because the targeting specificity is not precise enough, the resulting adverse effects are a concern and more specific drug targets urgently need to be developed [53-56]. The CTA NY-ESO-1, a homologous family member of TMEFF1, can induce a higher antibody titer and $\mathrm{CD}^{+} / \mathrm{CD}^{+} \mathrm{T}$ cell immune response, with a vaccine treatment prolonging the progression-free survival of patients with advanced ovarian cancer. It will thus be possible to develop monoclonal immunosuppressive agents that induce specific immune responses [57]. As an upstream factor of the MAPK or PI3K pathway, the high expression and carcinogenesis of TMEFF1 in endometrial carcinoma may have potential for the diagnosis of diseases, monitoring of cancer progression, and immunotargeted therapy, and become a new target for the treatment of endometrial carcinoma.

\section{Conclusions}

Based on the results of the current study, we confirmed that TMEFF1 plays an important role in endometrial carcinoma. As an independent prognostic factor for endometrial carcinoma, TMEFF1 promotes the invasion and migration of endometrial carcinoma cells, activates the PI3K/AKT and MAPK signaling pathways, and participates in the regulation of EMT. It may be useful as a prognostic biomarker and therapeutic target.

\section{Abbreviations}

UCEC: uterine corpus endometrial carcinoma; ChIP: chromatin immunoprecipitation assay; GO: Gene Ontology; KEGG: Kyoto Encyclopedia of Genes and Genomes; EMT: epithelial-to-mesenchymal; CTAs: Cancer/testis antigens; FIGO: International 
Federation of Gynecology and Obstetrics; ER: estrogen receptor; PR: progesterone receptor; $\mathrm{LN}$ : lymph node; SDS-PAGE: sodium dodecyl sulfatepolyacrylamide gel electrophoresis.

\section{Supplementary Material}

Supplementary tables.

http://www.jcancer.org/v12p5772s1.pdf

Supplementary table $\mathrm{S} 7$.

http:/ / www.jcancer.org/v12p5772s2.xlsx

\section{Acknowledgements}

\section{Ethics approval and consent to participate}

The experimental protocol was established, according to the ethical guidelines of the Helsinki Declaration and was approved by the Human Ethics Committee of China Medical University.

\section{Funding}

This work was supported by the National Natural Science Foundation of China [No. 81672590, 81602438]; Shengjing Free Researcher Project [No. 201804].

\section{Availability of data and materials}

The datasets used and analyzed during the current study are available from the corresponding author on reasonable request.

\section{Competing Interests}

The authors have declared that no competing interest exists.

\section{References}

1. Bray F, Ferlay J, Soerjomataram I, Siegel RL, Torre LA, Jemal A. Global cancer statistics 2018: GLOBOCAN estimates of incidence and mortality worldwide for 36 cancers in 185 countries. CA: a cancer journal for clinicians. 2018; 68: 394-424.

2. Bokhman JV. Two pathogenetic types of endometrial carcinoma. Gynecologic oncology. 1983; 15: 10-7.

3. Randall ME, Filiaci VL, Muss H, Spirtos NM, Mannel RS, Fowler J, et al. Randomized phase III trial of whole-abdominal irradiation versus doxorubicin and cisplatin chemotherapy in advanced endometrial carcinoma: a Gynecologic Oncology Group Study. Journal of clinical oncology : official journal of the American Society of Clinical Oncology. 2006; 24: 36-44

4. Salmaninejad A, Zamani MR, Pourvahedi M, Golchehre Z, Hosseini Bereshneh A, Rezaei N. Cancer/Testis Antigens: Expression, Regulation, Tumor Invasion, and Use in Immunotherapy of Cancers. Immunological investigations. 2016; 45: 619-40.

5. Grizzi F, Mirandola L, Qehajaj D, Cobos E, Figueroa JA, Chiriva-Internati M. Cancer-testis antigens and immunotherapy in the light of cancer complexity. International reviews of immunology. 2015; 34: 143-53.

6. Sammut SJ, Feichtinger J, Stuart N, Wakeman JA, Larcombe L, McFarlane RJ. A novel cohort of cancer-testis biomarker genes revealed through meta-analysis of clinical data sets. Oncoscience. 2014; 1: 349-59.

7. Condomines M, Hose D, Reme T, Requirand G, Hundemer M, Schoenhals M, et al. Gene expression profiling and real-time PCR analyses identify novel potential cancer-testis antigens in multiple myeloma. Journal of immunology (Baltimore, Md : 1950). 2009; 183: 832-40.

8. Gery S, Yin D, Xie D, Black KL, Koeffler HP. TMEFF1 and brain tumors. Oncogene. 2003; 22: 2723-7.

9. Laffaire J, Everhard S, Idbaih A, Criniere E, Marie Y, de Reynies A, et al. Methylation profiling identifies 2 groups of gliomas according to their tumorigenesis. Neuro-oncology. 2011; 13: 84-98.
10. Nie X, Liu C, Guo Q, Zheng MJ, Gao LL, Li X, et al. TMEFF1 overexpression and its mechanism for tumor promotion in ovarian cancer. Cancer management and research. 2019; 11: 839-55.

11. Rhodes DR, Kalyana-Sundaram S, Mahavisno V, Varambally R, Yu J, Briggs $\mathrm{BB}$, et al. Oncomine 3.0: genes, pathways, and networks in a collection of 18,000 cancer gene expression profiles. Neoplasia (New York, NY). 2007; 9: 166-80.

12. Chandrashekar DS, Bashel B, Balasubramanya SAH, Creighton CJ, Ponce-Rodriguez I, Chakravarthi B, et al. UALCAN: A Portal for Facilitating Tumor Subgroup Gene Expression and Survival Analyses. Neoplasia (New York, NY). 2017; 19: 649-58.

13. Vasaikar SV, Straub P, Wang J, Zhang B. LinkedOmics: analyzing multi-omics data within and across 32 cancer types. Nucleic acids research. 2018; 46: D956-d63.

14. Liberzon A, Subramanian A, Pinchback R, Thorvaldsdóttir H, Tamayo P, Mesirov JP. Molecular signatures database (MSigDB) 3.0. Bioinformatics (Oxford, England). 2011; 27: 1739-40.

15. Zhou Y, Zhou B, Pache L, Chang M, Khodabakhshi AH, Tanaseichuk O, et al. Metascape provides a biologist-oriented resource for the analysis of systems-level datasets. Nature communications. 2019; 10: 1523.

16. Warde-Farley D, Donaldson SL, Comes O, Zuberi K, Badrawi R, Chao P, et al. The GeneMANIA prediction server: biological network integration for gene prioritization and predicting gene function. Nucleic acids research. 2010; 38: W214-20.

17. Szklarczyk D, Gable AL, Lyon D, Junge A, Wyder S, Huerta-Cepas J, et al. STRING v11: protein-protein association networks with increased coverage, supporting functional discovery in genome-wide experimental datasets. Nucleic acids research. 2019; 47: D607-d13.

18. Tang Z, Li C, Kang B, Gao G, Li C, Zhang Z. GEPIA: a web server for cancer and normal gene expression profiling and interactive analyses. Nucleic acids research. 2017; 45: W98-w102.

19. Cerami E, Gao J, Dogrusoz U, Gross BE, Sumer SO, Aksoy BA, et al. The cBio cancer genomics portal: an open platform for exploring multidimensional cancer genomics data. Cancer discovery. 2012; 2: 401-4.

20. Saso S, Chatterjee J, Georgiou E, Ditri AM, Smith JR, Ghaem-Maghami S. Endometrial cancer. BMJ (Clinical research ed). 2011; 343: d3954.

21. Arend RC, Jones BA, Martinez A, Goodfellow P. Endometrial cancer: Molecular markers and management of advanced stage disease. Gynecologic oncology. 2018; 150: 569-80.

22. Azueta A, Gatius S, Matias-Guiu X. Endometrioid carcinoma of the endometrium: pathologic and molecular features. Seminars in diagnostic pathology. 2010; 27: 226-40.

23. Eib DW, Martens GJ. A novel transmembrane protein with epidermal growth factor and follistatin domains expressed in the hypothalamo-hypophysial axis of Xenopus laevis. Journal of neurochemistry. 1996; 67: 1047-55.

24. Eib DW, Holling TM, Zwijsen A, Dewulf N, de Groot E, van den Eijnden-van Raaij AJ, et al. Expression of the follistatin/EGF-containing transmembrane protein M7365 (tomoregulin-1) during mouse development. Mech Dev. 2000; 97: 167-71.

25. Noelker C, Schwake M, Balzer-Geldsetzer M, Bacher M, Popp J, Schlegel J, et al. Differentially expressed gene profile in the 6-hydroxy-dopamine-induced cell culture model of Parkinson's disease. Neuroscience letters. 2012; 507: 10-5.

26. Siegel DA, Huang MK, Becker SF. Ectopic dendrite initiation: CNS pathogenesis as a model of CNS development. International journal of developmental neuroscience : the official journal of the International Society for Developmental Neuroscience. 2002; 20: 373-89.

27. Pan Z, Tang B, Hou Z, Zhang J, Liu H, Yang Y, et al. XAGE-1b expression is associated with the diagnosis and early recurrence of hepatocellular carcinoma. Molecular and clinical oncology. 2014; 2: 1155-9.

28. Hussein YM, Ghareib AF, Mohamed RH, Radwan MI, Elsawy WH. MAGE-3 and MAGE-4 genes as possible markers for early detection of metastases in hepatitis $\mathrm{C}$ virus Egyptian patients complicated by hepatocellular carcinoma. Medical oncology (Northwood, London, England). 2012; 29: 994-9.

29. Ge W, Hu H, Ding K, Sun L, Zheng S. Protein interaction analysis of ST14 domains and their point and deletion mutants. The Journal of biological chemistry. 2006; 281: 7406-12

30. Gupta GP, Massague J. Cancer metastasis: building a framework. Cell. 2006; 127: 679-95.

31. Scheel C, Weinberg RA. Cancer stem cells and epithelial-mesenchymal transition: concepts and molecular links. Seminars in cancer biology. 2012; 22: 396-403.

32. Jayson GC, Kerbel $R$, Ellis LM, Harris AL. Antiangiogenic therapy in oncology: current status and future directions. Lancet (London, England). 2016; 388: 518-29.

33. De Craene B, Berx G. Regulatory networks defining EMT during cancer initiation and progression. Nature reviews Cancer. 2013; 13: 97-110.

34. Arano T, Fujisaki S, Ikemoto MJ. Identification of tomoregulin-1 as a novel addicsin-associated factor. Neurochemistry international. 2014; 71: 22-35.

35. Yang $\mathrm{P}$, Huo Z, Liao $\mathrm{H}$, Zhou Q. Cancer/testis antigens trigger epithelial-mesenchymal transition and genesis of cancer stem-like cells. Current pharmaceutical design. 2015; 21: 1292-300.

36. Moreno-Bueno G, Cubillo E, Sarrio D, Peinado H, Rodriguez-Pinilla SM, Villa $S$, et al. Genetic profiling of epithelial cells expressing E-cadherin repressors reveals a distinct role for Snail, Slug, and E47 factors in epithelial-mesenchymal transition. Cancer research. 2006; 66: 9543-56. 
37. Bolos V, Peinado H, Perez-Moreno MA, Fraga MF, Esteller M, Cano A. The transcription factor Slug represses E-cadherin expression and induces epithelial to mesenchymal transitions: a comparison with Snail and E47 repressors. Journal of cell science. 2003; 116: 499-511.

38. Stracquadanio G, Wang X, Wallace MD, Grawenda AM, Zhang P, Hewitt J, et al. The importance of p53 pathway genetics in inherited and somatic cancer genomes. Nature reviews Cancer. 2016; 16: 251-65.

39. Bell DW, Ellenson LH. Molecular Genetics of Endometrial Carcinoma. Annual review of pathology. 2019; 14: 339-67.

40. Ronneberg JA, Fleischer $\mathrm{T}$, Solvang $\mathrm{HK}$, Nordgard $\mathrm{SH}$, Edvardsen $\mathrm{H}$, Potapenko I, et al. Methylation profiling with a panel of cancer related genes: association with estrogen receptor, TP53 mutation status and expression subtypes in sporadic breast cancer. Molecular oncology. 2011; 5: 61-76.

41. Kauppinen JM, Kosma VM, Soini Y, Sironen R, Nissinen M, Nykopp TK, et al. ST14 gene variant and decreased matriptase protein expression predict poor breast cancer survival. Cancer epidemiology, biomarkers \& prevention : a publication of the American Association for Cancer Research, cosponsored by the American Society of Preventive Oncology. 2010; 19: 2133-42.

42. Harms PW, Chang C. Tomoregulin-1 (TMEFF1) inhibits nodal signaling through direct binding to the nodal coreceptor Cripto. Genes \& development. 2003; 17: 2624-9.

43. Chang C, Eggen BJ, Weinstein DC, Brivanlou AH. Regulation of nodal and BMP signaling by tomoregulin-1 (X7365) through novel mechanisms. Developmental biology. 2003; 255: 1-11.

44. Oshimori N, Fuchs E. The harmonies played by TGF-beta in stem cell biology. Cell stem cell. 2012; 11: 751-64.

45. Bao D, Lu D, Liu N, Dong W, Lu YD, Qin C, et al. Tomoregulin-1 prevents cardiac hypertrophy after pressure overload in mice by inhibiting TAK1-JNK pathways. Disease models \& mechanisms. 2015; 8: 795-804.

46. Zhu X, Kwon CH, Schlosshauer PW, Ellenson LH, Baker SJ. PTEN induces G(1) cell cycle arrest and decreases cyclin D3 levels in endometrial carcinoma cells. Cancer research. 2001; 61: 4569-75.

47. Wang J, Li Z, Wang X, Ding Y, Li N. The tumor suppressive effect of long non-coding RNA FRMD6-AS2 in uteri corpus endometrial carcinoma. Life sciences. 2020; 243: 117254

48. Rahman MA, Krainer AR, Abdel-Wahab O. SnapShot: Splicing Alterations in Cancer. Cell. 2020; 180: 208-.e1.

49. Skog J, Würdinger T, van Rijn S, Meijer DH, Gainche L, Sena-Esteves M, et al. Glioblastoma microvesicles transport RNA and proteins that promote tumour growth and provide diagnostic biomarkers. Nature cell biology. 2008; 10: $1470-6$

50. Wee P, Wang Z. Epidermal Growth Factor Receptor Cell Proliferation Signaling Pathways. Cancers. 2017; 9:52.

51. Leng X, Mei J, Liu L. [Molecular Mechanism of Different Signaling Pathways in Regulating PD-L1 Expression in EGFR Mutated Lung Adenocarcinoma]. Zhongguo fei ai za zhi = Chinese journal of lung cancer. 2018; 21: 875-9.

52. Uchida T, Wada K, Akamatsu T, Yonezawa M, Noguchi H, Mizoguchi A, et al. A novel epidermal growth factor-like molecule containing two follistatin modules stimulates tyrosine phosphorylation of erbB-4 in MKN28 gastric cancer cells. Biochemical and biophysical research communications. 1999; 266: 593-602.

53. Tortora G, Bianco R, Daniele G, Ciardiello F, McCubrey JA, Ricciardi MR, et al. Overcoming resistance to molecularly targeted anticancer therapies: Rational drug combinations based on EGFR and MAPK inhibition for solid tumours and haematologic malignancies. Drug resistance updates : reviews and commentaries in antimicrobial and anticancer chemotherapy. 2007; 10: 81-100.

54. Santarpia L, Lippman SM, El-Naggar AK. Targeting the MAPK-RAS-RAF signaling pathway in cancer therapy. Expert opinion on therapeutic targets. 2012; 16: 103-19.

55. Asati V, Mahapatra DK, Bharti SK. PI3K/Akt/mTOR and Ras/Raf/MEK/ERK signaling pathways inhibitors as anticancer agents: Structural and pharmacological perspectives. European journal of medicinal chemistry. 2016; 109: 314-41.

56. Chen X, Gao B, Ponnusamy M, Lin Z, Liu J. MEF2 signaling and human diseases. Oncotarget. 2017; 8: 112152-65.

57. Odunsi K, Matsuzaki J, Karbach J, Neumann A, Mhawech-Fauceglia P, Miller A, et al. Efficacy of vaccination with recombinant vaccinia and fowlpox vectors expressing NY-ESO-1 antigen in ovarian cancer and melanoma patients. Proceedings of the National Academy of Sciences of the United States of America. 2012; 109: 5797-802. 